\title{
A dynamic degradome landscape on miRNAs and their predicted targets in sugarcane caused by Sporisorium scitamineum stress
}

Yachun Su ${ }^{1,2}$, Xinhuan Xiao ${ }^{1}$, Hui Ling ${ }^{1}$, Ning Huang ${ }^{1}$, Feng Liu', Weihua Su', Yuye Zhang ${ }^{1}$, Liping Xu', Khushi Muhammad ${ }^{3}$ and Youxiong Que (,2* $^{1,}$

\begin{abstract}
Background: Sugarcane smut is a fungal disease caused by Sporisorium scitamineum. Cultivation of smut-resistant sugarcane varieties is the most effective way to control this disease. The interaction between sugarcane and $S$. scitamineum is a complex network system. However, to date, there is no report on the identification of microRNA (miRNA) target genes of sugarcane in response to smut pathogen infection by degradome technology.

Results: TaqMan qRT-PCR detection and enzyme activity determination showed that $S$. scitamineum rapidly proliferated and incurred significant enzyme activity changes in the reactive oxygen species metabolic pathway and phenylpropanoid metabolic pathway at $2 \mathrm{~d}$ and $5 \mathrm{~d}$ after inoculation, which was the best time points to study target gene degradation during sugarcane and S. scitamineum interaction. A total of $122.33 \mathrm{Mb}$ of raw data was obtained from degradome sequencing analysis of YC05-179 (smut-resistant) and ROC22 (smut-susceptible) after inoculation. The Q30 of each sample was >93\%, and the sequence used for degradation site analysis exactly matched the sugarcane reference sequence. A total of 309 target genes were predicted in sugarcane, corresponding to 97 known miRNAs and 112 novel miRNAs, and 337 degradation sites, suggesting that miRNAs can efficiently direct cleavage at multiple sites in the predicted target mRNAs. Gene Ontology (GO) annotation and Kyoto Encyclopedia of Genes and Genomes (KEGG) pathway analysis indicated that the predicted target genes were involved in various regulatory processes, such as signal transduction mechanisms, inorganic ion transport and metabolism, defense mechanisms, translation, posttranslational modifications, energy production and conversion, and glycerolipid metabolism. qRT-PCR analysis of the expression level of 13 predicted target genes and their corresponding miRNAs revealed that there was no obvious negative regulatory relationship between miRNAs and their target genes. In addition, a number of putative resistance-related target genes regulated by miRNA-mediated cleavage were accumulated in sugarcane during S. scitamineum infection, suggesting that feedback regulation of miRNAs may be involved in the response of sugarcane to $S$. scitamineum infection.
\end{abstract}

Conclusions: This study elucidates the underlying response of sugarcane to S. scitamineum infection, and also provides a resource for miRNAs and their predicted target genes for smut resistance improvement in sugarcane.

Keywords: Degradome, Sugarcane, Sporisorium scitamineum, Mycelial growth, Physiological index, miRNAs, miRNA target genes

\footnotetext{
* Correspondence: queyouxiong@126.com

'Key Laboratory of Sugarcane Biology and Genetic Breeding, Ministry of

Agriculture, Fujian Agriculture and Forestry University, Fuzhou 350002, China

${ }^{2}$ Key Laboratory of Ministry of Education for Genetics, Breeding and Multiple

Utilization of Crops, College of Crop Science, Fujian Agriculture and Forestry

University, Fuzhou 350002, China

Full list of author information is available at the end of the article
}

(c) The Author(s). 2019 Open Access This article is distributed under the terms of the Creative Commons Attribution 4.0 International License (http://creativecommons.org/licenses/by/4.0/), which permits unrestricted use, distribution, and reproduction in any medium, provided you give appropriate credit to the original author(s) and the source, provide a link to the Creative Commons license, and indicate if changes were made. The Creative Commons Public Domain Dedication waiver (http://creativecommons.org/publicdomain/zero/1.0/) applies to the data made available in this article, unless otherwise stated. 


\section{Background}

Sugarcane smut, caused by Sporisorium scitamineum, is a worldwide airborne fungal disease that affects sugarcane production [1]. The disease leads to severe losses in cane yield and reduces sucrose content and quality, thereby preventing further development of the sugarcane industry in China. A typical symptom of sugarcane smut involves cane tips of infected plants growing a black whip that points downwards and curls inwards around $120 \mathrm{~d}$ of planting. Simultaneously, mycelia invade the cane buds, and chlamydospores fall to the soil, thereby also infect sugarcane in the next growing season [2]. Compared to normal plants, the main stems of plants infected by $S$. scitamineum are small, the cane leaves are slender and light green in color, and tillers usually also grow black whips that lead to a sharp decline in sugarcane production [3]. Cultivating sugarcane varieties with excellent smut-resistance is the most economical and effective way to control the disease [4].

Due to the incomplete genome sequencing of Saccharum spp. hybrid, related genomics research is limited, thus hindering the progress of molecular improvement of sugarcane varieties. Current researches on the molecular mechanism of interaction between sugarcane and $S$. scitamineum mainly focus on genomics of smut pathogen [5-7], sugarcane molecular marker-assisted selection $[8,9]$, transcriptome $[10,11]$ and proteomic $[12,13]$ analysis, and resistance-related gene mining [10, 11]. The understanding of how miRNAs regulate the expression of their target genes in response to S. scitamineum infection is limited. The only earlier investigation relating to this matter was performed by our research group, which involved the identification of differentially expressed miRNAs in sugarcane challenged with S. scitamineum by using high-throughput sequencing [14].

MicroRNAs (miRNAs) are single-stranded, non-coding RNA molecules of approximately 21-24 nt in length in vivo [15]. miRNAs were first reported in Caenorhabditis elegans by Lee et al. [16]. Plant miRNAs were first obtained from a small Arabidopsis thaliana library by Reinhart et al. [17, 18]. miRNAs are encoded by endogenous miRNA genes and negatively regulate gene expression primarily through the degradation of target mRNAs during post-transcriptional gene silencing (PTGS) $[19,20]$. miRNAs are important regulators of organisms and are commonly involved in the response of plants to biotic stress [21, 22]. Previous studies have shown that plant miRNAs enhance their resistance to pathogen infection by regulating the expression of key disease-resistance genes [22-25]. During the late stage of wheat stripe rust infection, wheat miR408 was downregulated, and the expression of target gene chemocyanin-like protein (CLP1) was induced, which in turn inhibited the growth of mycelia in leaf [22]. After inoculation of tomato stalks with cucumber mosaic virus (CMV) and tomato aspermy virus (TAV), miR156 was accumulated, resulting in hollow and fibrotic stalks [23]. In addition, tomato plants overexpressing miR156 showed phenotypic symptoms that are similar to that of pathogen infections, indicating that miR156 regulates the interaction of tomato with pathogens [24]. miRNAs were differentially expressed between maize varieties with high resistance and susceptibility to Rhizoctonia solani [25]. The expression patterns of zea-miR168a, miR-2, and miR-6 were generally upregulated in diseaseresistant plants, while miR-3 was only upregulated in pathogen-infected sites [25]. The expression level of miRNA (except for miR-4 and miR-5) in Zea mays resistant variety was significantly higher than that in susceptible one, which induced host defense mechanisms to resist pathogen infection [25].

In organisms, the most important regulatory mechanism of miRNAs involves the miRNA-directed target cleavage that regulate their life cycle [26, 27]. Degradome sequencing, also called parallel analysis of RNA ends (PARE), is a high-throughput sequencing technique [28, 29]. This method can determine pairing information with miRNA through high-throughput deep sequencing of miRNA-mediated target gene cleavage degradation fragments, and the target genes of miRNAs are screened to determine how they regulate plant life activities in specific environments $[28,29]$. Degradome sequencing has been extensively applied to the identification of miRNA target genes in crops such as wheat [28], maize [30], peanut [31], and other crops. However, due to different calculation methods and screening criteria, it is difficult to avoid false positive in degradome sequencing data. Therefore, further validation of the authenticity and reliability of the miRNAs and their target genes is needed. Previous studies have shown that qRT-PCR can effectively detect the abundance of target genes in samples and verify the reliability of sequencing results in the degradome [32]. In addition, the qRT-PCR method can also be used to detect the expression level of the corresponding miRNAs, and then verify the interaction mode between miRNAs and their target genes [33].

In China, the main sugarcane variety grown during the past 20 years is ROC22, which is a Saccharum spp. hybrid from ROC5 × 69-463 (high-sugar line) and encompasses approximately $60 \%$ of the total sugarcane cultivated area. ROC22 is susceptible to S. scitamineum and results in a poor ratoon performance. In this study, an intergeneric $\mathrm{BC} 2$ hybrid with smut resistant character named YC05-179 (YC01-134 × ROC20) and the smut-susceptible variety ROC22 were used as experimental materials. First, the TaqMan qRT-PCR technology and physiological enzyme activity determination were performed to analyze the proliferation of $S$. scitamineum and activity changes of key enzymes involved in reactive oxygen species metabolic and phenylpropanoid metabolic pathways at 
various time points in different sugarcane genotypes infected by $S$. scitamineum. And the critical time points during interaction between sugarcane and S. scitamineum were determined. Second, the RNA of YC05-179 and ROC22 at different stages of infection was used for degradome sequencing analysis to screen miRNAs and their predicted target genes. Third, the expression level of partial obtained miRNAs and their corresponding predicted target genes was verified by qRT-PCR. The purpose of this study is to understand the miRNA-mediated molecular mechanisms in sugarcane response to S. scitamineum stress.

\section{Methods}

\section{Plant materials and inoculation}

The tested sugarcane varieties were YC05-179 (smut resistant) and ROC22 (smut susceptible). The pathogen strain was mixed spores of $S$. scitamineum collected from different sugarcane varieties and different planting areas in the field. They were all provided by the Key Laboratory of Sugarcane Biology and Genetic Breeding, Ministry of Agriculture, Fujian Agriculture and Forestry University (Fuzhou, Fujian, China). The plants were treated with S. scitamineum using the method of $\mathrm{Su}$ et al. [14]. The consistent and robust cane stems were selected, which were cut into single-bud stem, and then soaked in running water for $2 \mathrm{~d}$. The cane stems were then placed on a tray in a $32{ }^{\circ} \mathrm{C}$ incubator $(65 \%$ relative humidity) with $12 \mathrm{~h}$ light $/ 12 \mathrm{~h}$ dark conditions for germination. The water was replaced once in the morning and in the evening. When the cane buds had grown to about 2 $\mathrm{cm}$ in height, the treatment group were subjected to acupuncture inoculation with $5 \times 10^{6}$ spores $/ \mathrm{mL}$ S. scitamineum spore suspension (0.01\% Tween-20) in buds, and the control group underwent acupuncture inoculation with sterile water $(0.01 \%$ Tween-20). The cane stems were then placed in a $28^{\circ} \mathrm{C}$ incubator (65\% relative humidity) with $12 \mathrm{~h}$ light $/ 12 \mathrm{~h}$ dark conditions. The sugarcane bud tissues were collected at $0,1,2,3,5$ and $7 \mathrm{~d}$ after inoculation, fixed in liquid nitrogen, and then stored at $-80^{\circ} \mathrm{C}$ until analysis.

\section{Sugarcane genomic DNA and total RNA extraction and quality testing}

Three sugarcane buds were taken from each sample. Genomic DNA of the samples was extracted according to the modified CTAB method of Yao et al. [34]. Total RNA was extracted using TRIzol (Invitrogen, Carlsbad, CA, USA). The integrity of total RNA was detected by $1.5 \%$ agarose gel electrophoresis. The concentration and purity of genomic DNA and total RNA were assessed using a Nano-Drop (Thermo Fisher, USA) and Agilent 2100 (Agilent Technologies, Palo Alto, CA, USA) systems.

\section{Quantification of smut pathogen in sugarcane}

The quantification of S. scitamineum in YC05-179 and ROC22 was detected using TaqMan qRT-PCR technology that was developed by Su et al. [35]. The detection primers were bEQ-F: 5'-TGAAAGTTCTCATGCAAGCC-3' and bEQ-R: 5'-TGAGAGGTCGATTGAGGTTG-3', and the TaqMan probe was 5'-FAM-TGCTCGACGCCAATTCG GAG-TAMRA-3'. A standard curve was established by a 10 -fold gradient dilution with recombinant plasmid DNA containing the $b E$ gene (b East mating type gene, GenBank Accession No. U61290.1). The concentration of DNA was $500 \mathrm{ng} / \mu \mathrm{L}$. The amplification program was $50^{\circ} \mathrm{C}$ for $2 \mathrm{~min}$; followed by 40 cycles of $95^{\circ} \mathrm{C}$ for 10 min, $95^{\circ} \mathrm{C}$ for $15 \mathrm{~s}, 60^{\circ} \mathrm{C}$ for $1 \mathrm{~min}$; and a single-point fluorescence detection at $60^{\circ} \mathrm{C}$. The blank control replaces the template DNA with an equal volume of $\mathrm{ddH}_{2} \mathrm{O}$. The negative control is the DNA of pathogenfree FN41 (a newly released Saccharum spp. hybrid from Yuetang91-976 × ROC20 in China) 4-month-old plantlets and the positive control is the genomic DNA of S. scitamineum. Each run of TaqMan qRT-PCR contained three replicates. A standard curve was drawn using Microsoft Excel and GraphPad Prism softwares, and the quantification of S. scitamineum in each sample was calculated.

\section{Determination the activity of key enzymes involved in reactive oxygen species metabolic and phenylpropanoid metabolic pathways}

At $0,1,2,3,5$ and $7 \mathrm{~d}$ after inoculation, $0.1 \mathrm{~mol} / \mathrm{L}$ borate buffer ( $\mathrm{pH} 8.7$ ) was used for the extraction of crude enzyme solution in cane bud tissues [36]. All samples (five buds per sample) were weighed and homogenized with ice-cold borate buffer at the ratio of $1 \mathrm{~g} / 10 \mathrm{~mL}$, as well as with a small amount of quartz sand and polyvinylpyrrolidone (PVP). The supernatant was centrifuged at $5000 \mathrm{rpm} / \mathrm{min}$ for $15 \mathrm{~min}$ at $4{ }^{\circ} \mathrm{C}$. The final supernatant was used as the crude enzyme solution for the determination of peroxidase (POD), superoxide dismutase (SOD), catalase (CAT), polyphenol oxidase (PPO), phenylalanine ammonia lyase (PAL), and tyrosine ammonia lyase (TAL) activities. The guaiacol method was used to determine POD enzyme activity [37]. SOD activity was measured using nitroblue tetrazolium (NBT) photoreduction [38]. The activities of CAT, PPO, PAL, and TAL were determined according to the methods of Beers and Sizer [39], Galeazzi et al. [40], Green et al. [41], and Kofalvi and Nassuth [42], respectively. Three biological replicates were prepared for each treatment. The data were analyzed using Microsoft Excel and SPSS softwares. GraphPad Prism software was used to produce figures. Duncan's method was used for statistical analysis. 


\section{Degradome library construction, sequencing and bioinformatics analysis}

The samples for degradome sequencing were YC05-179 and ROC22 cane buds inoculated with sterile water at $0 \mathrm{~d}$ (control group) and with S. scitamineum at 2 and $5 \mathrm{~d}$ (treated group). These samples were named Y0, Y2, Y5, R0, R2, and R5, respectively. The quality of total RNA met the $\mathrm{OD}_{260 / 280}$ of 1.8-2.2, with normal absorption peak at $260 \mathrm{~nm}$, and $28 \mathrm{~S} / 18 \mathrm{~S}$ ratio $\geq 1.5$. Library construction, degradome sequencing, and data analysis were commissioned by Beijing BioMed Biotech Co., Ltd. (Beijing, China) using the Illumina $\mathrm{HiSeq}^{\mathrm{Tm}} 2500$ [43]. Raw reads were generated from degradome sequencing. Then the reads with adapters, the low-quality reads with mass values below 30 and bases in excess of $20 \%$, the reads with unknown base $\mathrm{N}$ contents greater than or equal to $10 \%$, and the reads less than $18 \mathrm{nt}$ in length were filtered out to eventually obtain clean reads and cluster reads [31]. Non-coding RNAs (rRNA, scRNA, snoRNA, snRNA, and tRNA) were removed by aligning clean reads and cluster reads with the Rfam database [44]. Because whole genome sequencing of Saccharum spp. hybrid has not been completed to date, we mapped the remaining sequences using the bowtie software and targetfinder software to align with the sugarcane reference sequences (sugarcane transcriptome under S. scitamineum stress [11], GSS database, and EST database in NCBI) and the known miRNAs from miRBase 21.0 (http://www.mirbase.org) or miRNAs identified in our previous study [14] to predict miRNA target genes. When the score of miRNA that matches the mRNA was less than or equal to 7 , the transcript sequence was considered as the miRNA target gene $[45,46]$.

\section{miRNA targets prediction and identification}

Based on the depth statistics of mRNA target genes and the abundance of transcripts, the target genes were grouped into five categories, namely, $0,1,2,3$, and 4 [45, 47]. Category 0 indicated that the position had a depth $>1$, an abundance equal to the maximum of the transcript abundance, and the transcript had only one maximum value. Category 1 indicated that the position had a depth $>1$, an abundance equal to the maximum value of the transcript abundance, and the transcript had two or more maxima. Category 2 represented the depth of the position was $>1$ and the abundance was less than the maximum but higher than the mean of the transcript abundance. Category 3 represented that the depth was $>1$ and the abundance was less than or equal to the mean of the transcript abundance. Category 4 represented the depth of the position equal to 1 .

The degradation site of predicted target gene was analyzed by Cleaveland software at $p$-value $<0.05$. The screened predicted target gene sequences with degradation sites were compared to the COG, GO, KEGG, NR, NT, and Swiss-Port databases to obtain the predicted target gene annotation information. The expression level of the predicted target gene was calculated using the fragments per kilobase of transcript per million mapped reads (FPKM) method which eliminated the influence of gene length and sequencing difference in high-throughput sequencing [48]. The treatment group and the control group were compared to analyze the differential expression of the predicted target genes, and the ratio of expression level was expressed as the fold-change [49]. A fold-change $\geq 2$ and a false discovery rate (FDR) $<0.01$ were used as screening criteria for differentially expressed predicted target genes. The FDR was obtained by correcting the difference in the significance of the $p$-value [50]. The $p$-value was corrected using the Benjamini-Hochberg calibration method, and the FDR was used as a screening index to ensure the quality of differentially expressed genes. DY2 and DY5 represent the differentially expressed predicted target genes at 2 and $5 \mathrm{~d}$ after YC05-179 was inoculated with S. scitamineum, whereas DR2 and DR5 represent the differentially expressed predicted target genes at 2 and $5 \mathrm{~d}$ after ROC22 was inoculated with S. scitamineum. DY2, DY5, DR2, and DR5 were respectively analyzed by COG, $\mathrm{GO}$, and KEGG to investigate functional and related metabolic pathways of the differentially expressed predicted target genes. The continuously and non-continuously differentially expressed target genes predicted in YC05179 and ROC22 at different time points were analyzed to reveal potential genes that were related to smut resistance.

\section{qRT-PCR validation of the expression level of miRNAs and their predicted targets}

MiR168a-5p, miR5293, miR160a, nov-miR132, nov-mir143, nov-mir-63, nov-mir-18, miR5368, nov-mir-10, miR858b, nov-mir-97, miR162a, miR529-3p, and their partial predicted target genes (Table 1 ) were verified by qRT-PCR. The qRT-PCR primers of the predicted target genes (Additional file 1: Table S1) were designed using Beacon Designer software. Glyceraldehyde-3-phosphate dehydrogenase $(G A P D H)$ was used as the internal reference gene [51]. Total RNA of YC05-179 and ROC22 inoculated with sterile water for $0 \mathrm{~d}$ and with S. scitamineum for 2 and $5 \mathrm{~d}$ were digested with RNase-Free DNase (Promega, USA) to remove DNA contamination, followed by reverse transcription to generate first-strand cDNA using a PrimeScript ${ }^{\oplus}$ RT reagent kit (Perfect Real Time) (TaKaRa, Dalian, China). The qRT-PCR reaction system was prepared using the SYBR Green dye method following the instructions of the FastStart Universal SYBR Green PCR Master (ROX) kit (Roche, Shanghai, China). qRT-PCR amplification was performed on an ABI 7500 instrument at $50{ }^{\circ} \mathrm{C}$ for $2 \mathrm{~min}$, followed by 40 cycles of $95^{\circ} \mathrm{C}$ for $15 \mathrm{~s}$ and $60^{\circ} \mathrm{C}$ for $1 \mathrm{~min}$. Three replicates were 
Table 1 The basic information of selected miRNAs and their corresponding predicted target genes for qRT-PCR validation

\begin{tabular}{llllll}
\hline No. & miRNA name & Predicted target gene ID & Predicted target gene annotation & Cleavage site & Category \\
\hline 1 & miR168a-5p & Sugarcane_Unigene_BMK.66779 & Protein argonaute 1B (AGO 1B) & 984 & 0 \\
2 & miR5293 & Sugarcane_Unigene_BMK.40335 & Auxin-induced protein (AIP) & 720 & 1 \\
3 & miR160a & Sugarcane_Unigene_BMK.63027 & Auxin response factor 8 (ARF8) & 879 & 0 \\
4 & nov-mir-132 & Sugarcane_Unigene_BMK.28594 & Cinnamoyl-CoA reductase (CCR) & 588 & 1 \\
5 & nov-mir-143 & Sugarcane_Unigene_BMK.64656 & Ethylene insensitive 3-like 3 protein (EIL3) & 1740 \\
6 & nov-mir-63 & Sugarcane_Unigene_BMK.75694 & Glycerol kinase (GK) & 1790 & 0 \\
7 & miR396e-5p & Sugarcane_Unigene_BMK.60551 & Growth-regulating factor 8 (GRF8) & 465 \\
8 & nov-mir-66 & Sugarcane_Unigene_BMK.60551 & Growth-regulating factor 8 (GRF8) & 465 \\
9 & nov-mir-18 & Sugarcane_Unigene_BMK.60551 & Growth-regulating factor 8 (GRF8) & 0 \\
10 & miR5368 & Sugarcane_Unigene_BMK.51989 & Hypersensitive-induced response protein 1 (HIR1) & 465 \\
11 & nov-mir-10 & Sugarcane_Unigene_BMK.62668 & Mildew resistance locus O (MLO) & 866 \\
12 & miR858b & gi35098237 & Myb-related protein Hv33 (MYB2) & 510 \\
13 & nov-mir-97 & Sugarcane_Unigene_BMK.51113 & Protein phosphatase 2C (PP2C) & 1 \\
14 & miR162a & Sugarcane_Unigene_BMK.67816 & S-adenosylmethionine decarboxylase (SAMDC) & 524 \\
15 & miR529-3p & Sugarcane_Unigene_BMK.64654 & Ubiquitin carboxyl-terminal hydrolase isozyme L5-like (UCH-L5) & 864 & 1 \\
\hline
\end{tabular}

Cleavage site, nucleotide number from 5 ' end of CDNA; Category, the "category" of this cleaveage site

used for each sample. The blank controls were used to replace the cDNA template with sterile water. Stemloop method was used to detect the expression level of candidate miRNA [52]. According to the method of Varkonyi-Gasic et al. [33], miRNA stem-loop primers (RT primer) and upstream primers (Additional file 1: Table S2) were designed. The downstream primer was a universal primer for anchoring the stem-loop region. $G A P D H$ was used as the internal reference gene [53]. Total RNA was reverse-transcribed using an Applied Biosystems ${ }^{\circ}$ TaqMan $^{\circ}$ MicroRNA Reverse Transcription kit (Invitrogen, Carlsbad, CA, USA) with a $15-\mu \mathrm{L}$ reverse-transcription system. In the process of reverse transcription, when using cDNA template as internal control, the RT primers were replaced by random primers. The miRNA qRT-PCR reaction system and procedure were the same as that for the predicted target gene. qRT-PCR data were calculated using the $2^{-\Delta \Delta \mathrm{CT}}$ method [54].

\section{Results}

Smut pathogen proliferation and changes in the activity of key enzymes involved in reactive oxygen species metabolic and phenylpropanoid metabolic pathways during the early stage of infection

The results of TaqMan qRT-PCR (Fig. 1) showed that the $\mathrm{Ct}$ values of smut-resistant genotype (YC05-179) and -susceptible genotype (ROC22) inoculated with sterile water, negative control, and blank control were all higher than 37, indicating the absence of S. scitamineum, whereas the $\mathrm{Ct}$ value of samples inoculated with $S$. scitamineum was between 27 and 33. The quantification of S. scitamineum in YC05-179 and ROC22 increased with inoculation time, which were $323,995.15 \pm 53,563.55$ copies/ $\mu \mathrm{L}-$ $2,935,184.09 \pm 36,789.33$ copies $/ \mu \mathrm{L}$ and $340,733.51 \pm$ $29,137.42$ copies $/ \mu \mathrm{L}-7,525,544.93 \pm 358,488.58$ copies $/ \mu \mathrm{L}$, respectively. At $0 \mathrm{~d}$ after inoculation, the quantification of S. scitamineum was similar in YC05-179 and ROC22. The dynamic increase in DNA copy number of the S. scitamineum in ROC22 was more distinct at 1, 2 and $3 \mathrm{~d}$ after

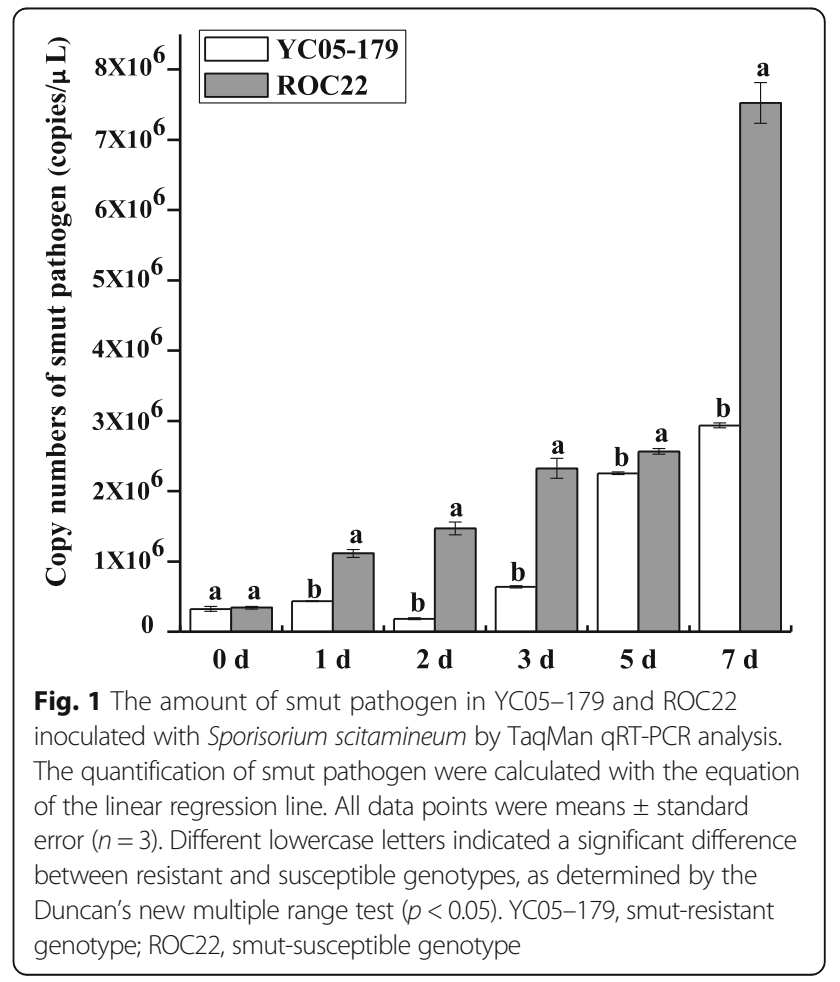


inoculation, which was 3.27-, 4.31-, and 6.82-fold respectively of that at $0 \mathrm{~d}$ after inoculation. The quantification of S. scitamineum in YC05-179 was increased at $1 \mathrm{~d}$ but decreased at $2 \mathrm{~d}$, which was 1.34 -fold and 0.57 -fold that at $0 \mathrm{~d}$, respectively. At 5 and $7 \mathrm{~d}$, the content of S. scitamineum in YC05-179 showed minimal change, which was 6.96- and 9.06-fold that at $0 \mathrm{~d}$, whereas the content of $S$. scitamineum in ROC22 continued to increase and peaked at $7 \mathrm{~d}$, which was 22.09-fold that at $0 \mathrm{~d}$ after inoculation.

Figure 2 showed the activity changes of six key enzymes involved in reactive oxygen species metabolic and phenylpropanoid metabolic pathways in two sugarcane genotypes post inoculation with $S$. scitamineum. After inoculation, the activity of POD in YC05-179 and ROC22 was all increased. It peaked at 2,3 and $5 \mathrm{~d}$ in YC05-179, whereas at 3 and $7 \mathrm{~d}$ in ROC22. The activity of SOD in YC05-179 was decreased at $1 \mathrm{~d}$ but gradually increased and reached a peak value at $7 \mathrm{~d}$. In ROC22, the activity of SOD was stable at $1 \mathrm{~d}$, and decreased at 2 and $7 \mathrm{~d}$, whereas reached a peak value at $5 \mathrm{~d}$. There was an opposite expression pattern in the activity of CAT between YC05-
179 and ROC22. After inoculation with S. scitamineum, the activity of CAT in YC05-179 was significantly increased and peaked at $1 \mathrm{~d}$, follow by 2,5 and $7 \mathrm{~d}$, whereas that in ROC22 was decreased at 1, 2 and $5 \mathrm{~d}$. PAL and TAL, which are upstream of the phenylpropanoid metabolic pathway, inhibit lignin biosynthesis when their activities are inhibited [55]. Inoculation of YC05-179 with S. scitamineum resulted in a stronger activity of PAL at $3 \mathrm{~d}$, followed by 2 and $5 \mathrm{~d}$. The activity of PAL in ROC22 was increased at 3 and $7 \mathrm{~d}$ but decreased at 2 and $5 \mathrm{~d}$. In YC05-179, the activity of TAL reached a peak value at 1 and $5 \mathrm{~d}$, followed by $3 \mathrm{~d}$, and remained stable at 2 and $7 \mathrm{~d}$. The activity of TAL in ROC22 were all increased after inoculation and peaked at $1,2,5$ and $7 \mathrm{~d}$. PPO, which catalyzes the phenols to quinones, was assumed to be involved in plant defense against pathogens [56]. After inoculation, the activity of PPO in YC05-179 was significantly increased and peaked at 1 , 2 and $7 \mathrm{~d}$, followed by 3 and $5 \mathrm{~d}$. The activity of PPO in ROC22 was significantly increased at 2 and $5 \mathrm{~d}$ but remained stable at the other time points. In summary, the activities of POD, SOD, CAT, PPO, PAL and TAL

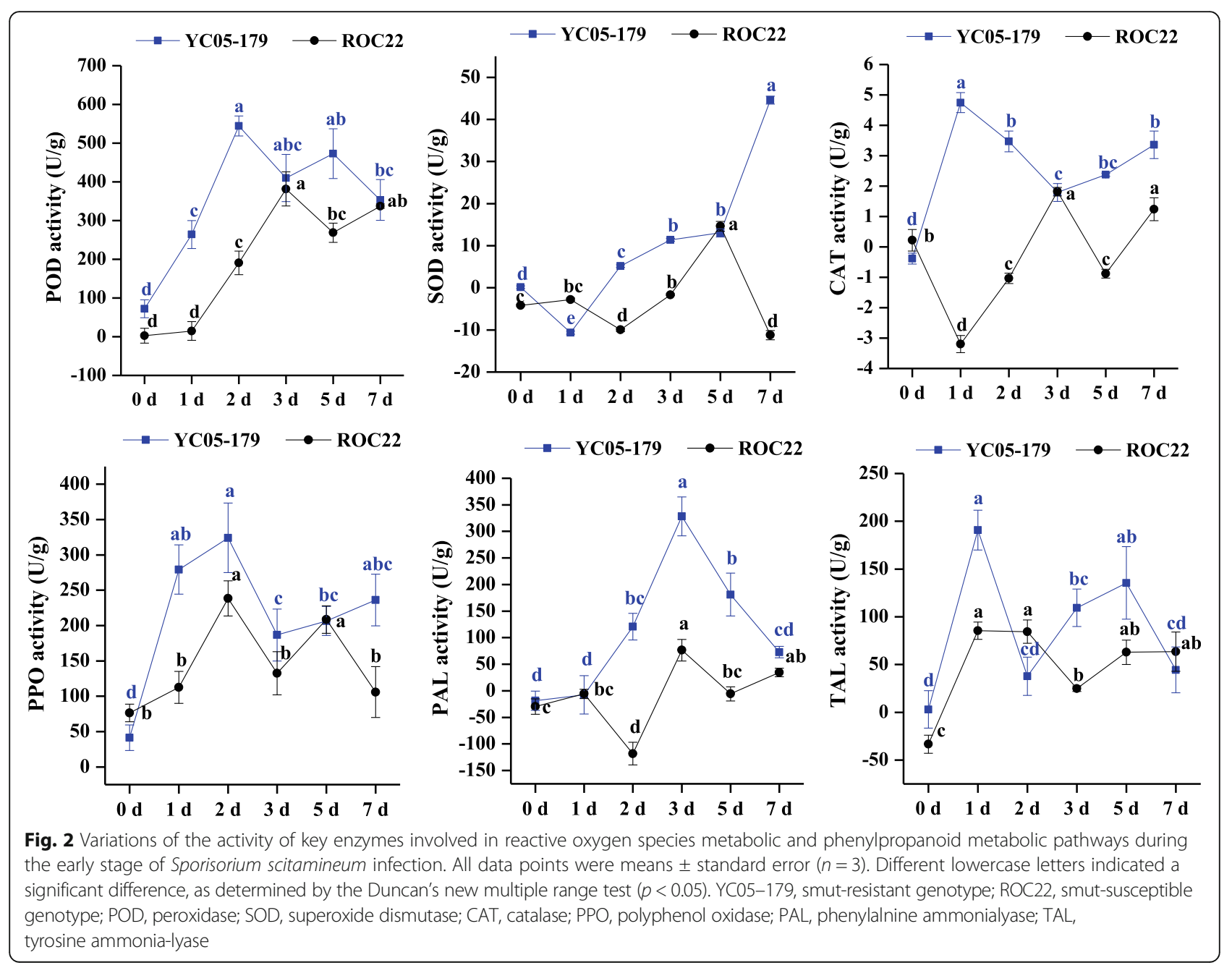


in YC05-179 were generally higher than those in the control (0 d) and ROC22 plants after inoculation with S. scitamineum. At 2 and $5 \mathrm{~d}$, the metabolic levels of activated oxygen and phenylpropanoid in YC05-179 were stronger than those in ROC22. Furthermore, there was a significant difference in smut pathogen content between YC05-179 and ROC22 after inoculation (Fig. 1). At the early stage of $2 \mathrm{~d}$, the amount of $S$. scitamineum was decreased in YC05-179 but increased in ROC22 (Fig. 1). Therefore, we used 2 and $5 \mathrm{~d}$ after inoculation with $S$. scitamineum as the best sampling time points for degradome sequencing.

\section{Degradome library construction and data summary}

Six cane-bud samples, including YC05-179 and ROC22 inoculated with sterilized water for $0 \mathrm{~d}$ and those inoculated with S. scitamineum for 2 and $5 \mathrm{~d}$, were sequenced. Each sample was generated with no less than $10 \mathrm{M}$ reads, and a total of $122.33 \mathrm{M}$ raw reads in six samples was gained. After data evaluation, clean reads and cluster reads were obtained with a length of $47 \mathrm{nt}$. The number of clean reads in the Y0, Y2, Y5, R0, R2, and R5 libraries was 18,662,178, 21,772,014, 22,928,020, 19,440,129, 18,537,103, and 20,995,053, respectively (Table 2). After comparing the clean reads and cluster tags with Rfam database to exclude rRNAs, tRNAs, snoRNAs, and snRNA (except for miRNAs), the remaining sequences were aligned with the reference sequence of sugarcane to obtain the fully mapped data, which consisted of 2,748,695 (Y0), 3,333,316 (Y2), 3,446,802 (Y5), 2,490,391 (R0), 3,151,567 (R2), and $3,001,075$ (R5) reads (Table 2) that were then used in the analysis of degradation sites.

\section{Degradation site specificity and diversity}

According to sequence homology, each miRNA can simultaneously target two or more target genes belonging to the same type or having similar conserved domains, and a target gene can also be cleaved by multiple miRNAs $[57,58]$. In this study, the degradome sequencing results showed that the predicted target gene could be cleaved by different miRNAs at a specific cleavage site. For example, Sugarcane_Unigene_BMK.74449 could be cleaved simultaneously by miR165a, miR166a, and miR166g-3p at position 4321 (Figs. 3a, b, c). The predicted target gene could also be cleaved by different miRNAs at different cleavage sites, e.g., Sugarcane_Unigene_BMK.61043 could be cleaved by nov-mir-84 and nov-mir- 41 at positions 324 and 785, respectively (Figs. 3d, e). In addition, multiple target genes could be cleaved by the same miRNA. For example, Sugarcane_Unigene_BMK.74449 and Sugarcane_Unigene_BMK.72615 could be cleaved by miR165a (Figs. 3a, f).

\section{Target gene identification, annotation, and classification}

Based on the miRNA database [14] and Unigene database [11] of sugarcane after smut pathogen infection, 2922 miRNA-mRNA pairs were screened from six libraries using targetfinder software. A total of 337 degradation sites were detected by Cleaveland software, corresponding to 219 miRNAs (97 known miRNAs and 112 new miRNAs) and 309 predicted target mRNAs (Additional file 2: Table S3). The predicted target mRNAs were all mainly classified as Category 0 in all six libraries, without Category 4 (Fig. 4). We found that the predicted target genes cleaved by miRNAs, such as squamosa promoter-binding-like protein $(S P L)$, no apical meristem $(N A M)$, v-myb avian myeloblastosis viral oncogene homolog $(M Y B)$, auxin response factor, extensin-like protein, somatic embryogenesis receptor kinase (SERK), CAT, ethylene-insensitive 3-like 3 protein (EIL3), and miRNA precursor encoding genes, may be involved in various life-controlling processes of sugarcane.

\section{COG, GO and KEGG analyses of differentially expressed predicted target genes}

Differentially expressed predicted target genes were screened according to the criteria of fold-change $\geq 2$ and FDR $<0.01$.

Table 2 Statistic results of degradome sequencing data

\begin{tabular}{|c|c|c|c|c|c|c|c|c|}
\hline Sample name & Total reads & Tags number & Tags percent & Clean reads & Q30 & $\begin{array}{l}\text { Clean reads after } \\
\text { Rfam alignment }\end{array}$ & Mapped data & Mapped data percentage \\
\hline Y0 & $18,665,458$ & 3280 & $0.02 \%$ & $18,662,178$ & $94.29 \%$ & $6,613,553$ & $2,748,695$ & $41.56 \%$ \\
\hline Y2 & $21,777,063$ & 5049 & $0.02 \%$ & $21,772,014$ & $94.45 \%$ & $8,270,381$ & $3,333,316$ & $40.30 \%$ \\
\hline Y5 & $22,944,082$ & 16,062 & $0.07 \%$ & $22,928,020$ & $94.59 \%$ & $8,609,667$ & $3,446,802$ & $40.03 \%$ \\
\hline RO & $19,451,881$ & 11,752 & $0.06 \%$ & $19,440,129$ & $93.73 \%$ & $6,342,529$ & $2,490,391$ & $39.26 \%$ \\
\hline R2 & $18,549,222$ & 12,119 & $0.07 \%$ & $18,537,103$ & $94.56 \%$ & $7,226,017$ & $3,151,567$ & $43.61 \%$ \\
\hline R5 & $20,999,522$ & 4469 & $0.02 \%$ & $20,995,053$ & $94.46 \%$ & $7,007,751$ & $3,001,075$ & $42.83 \%$ \\
\hline
\end{tabular}

Total reads, total raw reads of sequencing; Tags number, reads number with adaptor; Tags percent, proportion of reads with adaptors in total reads; Clean reads, clean reads numbers after filtering; Q30, percentage of Q30; Clean reads after Rfam alignment, reads numbers of samples compared with sugarcane reference sequences (sugarcane transcriptome under smut pathogen stress [11], GSS database, and EST database in NCBI); Mapped data, reads numbers of samples compared to sugarcane reference sequences; Mapped data percentage, percentage of mapped numbers in the clean reads after Rfam alignment. Y0 and R0 mean the genes in the libraries of YC05-179 and ROC22 inoculated with sterile water at $0 \mathrm{~d}$, respectively. $Y 2$ and $Y 5$ mean the genes in the libraries of YC05-179 inoculated with Sporisorium scitamineum at 2 and 5 d, respectively. R2 and R5 mean the genes in the libraries of ROC22 inoculated with S. scitamineum at 2 and 5 d, respectively. YC05-179, smut-resistant genotype; ROC22, smut-susceptible genotype 


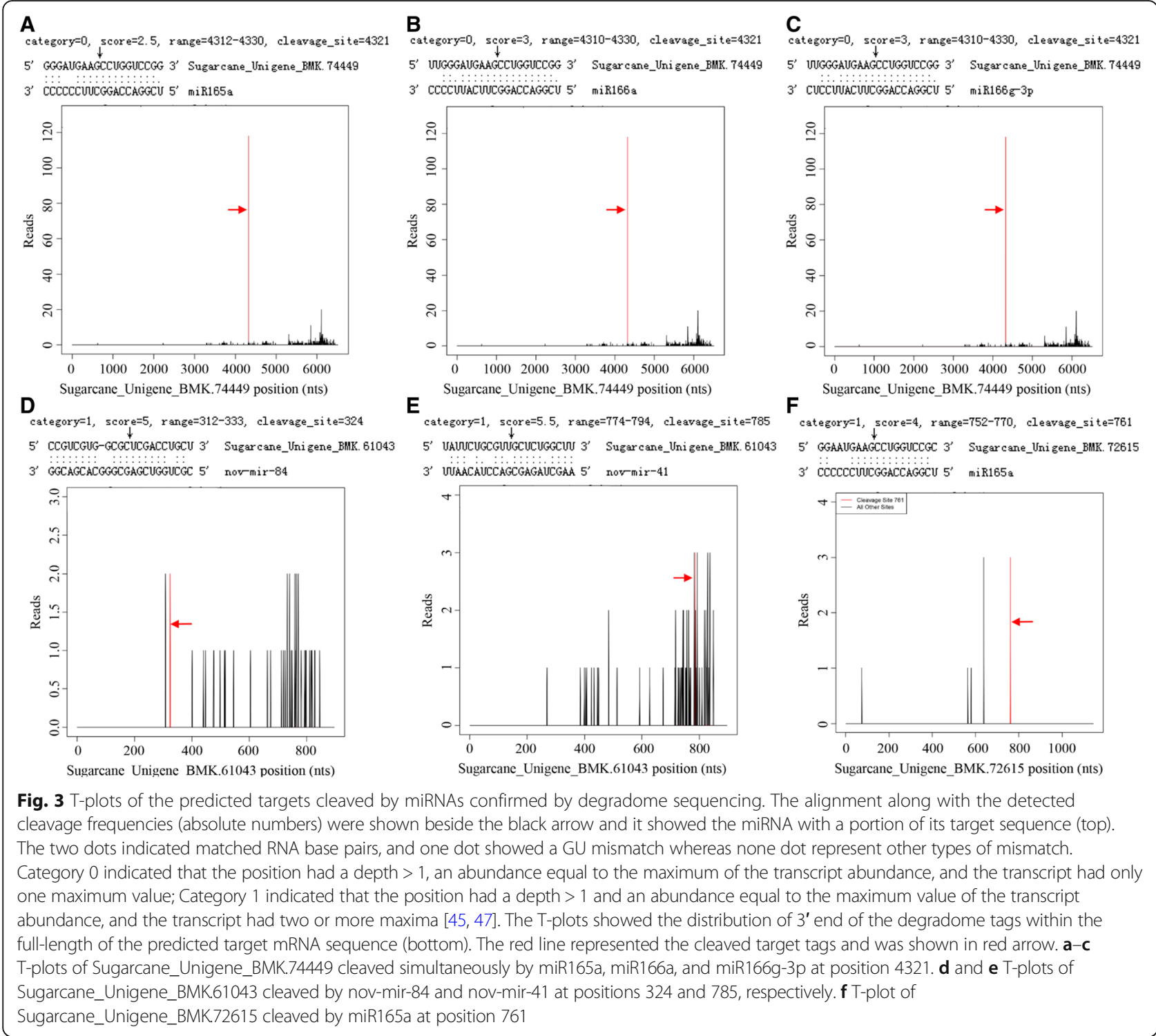

The results showed that 69 predicted target genes (31 upregulated and 38 downregulated) of DY2 and 73 predicted target genes (37 upregulated and 36 downregulated) of DY5 were differentially expressed in YC05-179. A total of 144 predicted target genes (70 upregulated and 74 downregulated) of DR2 and 138 predicted target genes (71 upregulated and 67 downregulated) of DR5 were differentially expressed in ROC22. In conclusion, the total number of upregulated predicted target genes was similar to that of downregulated predicted target genes at both 2 and $5 \mathrm{~d}$ in either YC05-179 or ROC22.

COG analysis showed that the differentially expressed predicted target genes in DY2, DY5, DR2 and DR5 were functionally annotated to the categories of signal transduction mechanisms $(1,0,2,0)$, energy production and conversion $(2,1,2,4)$, and inorganic ion transport and metabolism (1, 1, 2, 3), etc. (Additional file 1: Figure S1). GO enrichment results showed that the differentially expressed predicted target genes of DY2, DY5, DR2, and DR5 were involved in 28, 31, 31 and 33 GO categories, respectively (Table 3), of which highly enriched GO categories in terms of cellular function were cell $(35,33$, $85,85)$, membrane $(18,17,29,36)$, organelle $(30,31,79$, $80)$, and cell part $(35,33,85,85)$. GO categories that were highly enriched in terms of molecular function were catalytic activity $(20,12,39,40)$, and binding $(25$, $26,57,53)$. GO categories with more enriched biological process were metabolic process $(21,19,50,56)$, cellular process $(22,23,60,62)$, single-organism process $(21,18$, $47,49)$, response to stimulus $(12,8,27,30)$, and biological regulation $(16,12,34,35)$. 


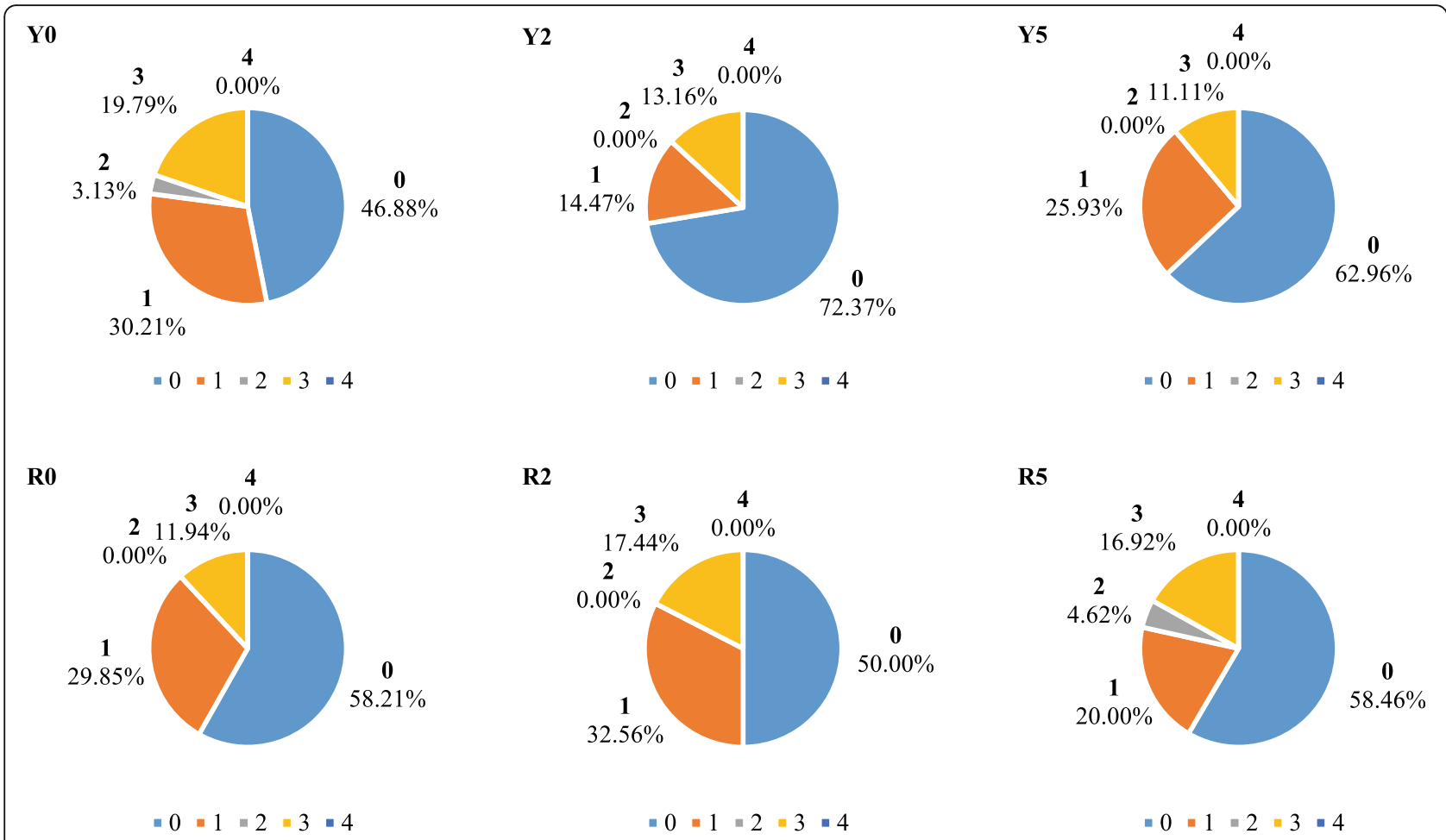

Fig. 4 The classification of the predicted target genes in the six libraries. Y0 and R0 mean the predicted target genes in the libraries of YC05-179 and ROC22 inoculated with sterile water at $0 \mathrm{~d}$, respectively. $\mathrm{Y} 2$ and $\mathrm{Y} 5$ mean the predicted target genes in the libraries of YC05-179 inoculated with Sporisorium scitamineum for $2 \mathrm{~d}$ and $5 \mathrm{~d}$, respectively. R2 and R5 mean the predicted target genes in the libraries of ROC22 inoculated with S. scitamineum for $2 \mathrm{~d}$ and $5 \mathrm{~d}$, respectively

Table 4 showed the results of KEGG pathway enrichment in YC05-179 and ROC22 post inoculation with $S$. scitamineum. The differentially expressed predicted target genes involved in disease resistance-related metabolic pathways in YC05-179 and ROC22 mainly belonged to the categories of plant hormone signal transduction, ubiquinone and other terpenoid-quinone biosynthesis, ubiquitin mediated proteolysis, plant-pathogen interaction, oxidative phosphorylation, peroxisome, phenylalanine, tyrosine and tryptophan biosynthesis, and phagosome. Among them, the differentially expressed predicted target genes involved in plant-pathogen interaction were only found in YC05-179, whereas the differentially expressed predicted target genes involved in phenylalanine, tyrosine, and tryptophan biosynthesis and phagosome were only found in ROC22.

\section{Identification of potential resistance-related target gene Continuous differential expression of predicted target genes common to YCO5-179 and ROC22}

Venn diagram analysis of DY2, DY5, DR2, and DR5 showed that there were 38 continuously differentially expressed predicted target genes in YC05-179 at 2-5 d after infection with S. scitamineum, of which 91 genes were in ROC22 (Fig. 5). Comparative analysis showed that 15 continuously differentially expressed predicted target genes were shared by $\mathrm{YC} 05-179$ and ROC22, such as Sugarcane_Unigene_BMK.64130 (transcription factor GAMYB), gi35264535 (E3 ubiquitin-protein ligase), Sugarcane_Unigene_BMK.51816 (Formin-like protein 5), Sugarcane_Unigene_BMK.52182 (protein argonaute 1D), Sugarcane_Unigene_BMK.51113 (protein phosphatase 2C, PP2C), and Sugarcane_Unigene_BMK.40335 (auxininduced protein), etc.. If these genes are involved in the pathogenicity-related pathways of plants, then they may be considered as candidate genes for smut-resistance research in the further.

\section{Continuous differential expression of predicted target genes specific to YC05-179 or ROC22}

Figure 5 showed that there were 12 predicted target genes that were differentially expressed only after YC05-179 was inoculated with $S$. scitamineum, namely, Sugarcane Unigene_BMK.45107 (Myb-related protein), gi36066484 (Aquaporin SIP1-2), Sugarcane_Unigene_BMK.75694 (glycerol kinase), Sugarcane_Unigene_BMK.52252 (cell wall-associated hydrolase), gi36009271 (nuclear pore complex protein NUP133), Sugarcane_Unigene_BMK.75849 
Table 3 Gene ontology based on differentially expressed predicted target genes

\begin{tabular}{|c|c|c|c|c|c|c|}
\hline \multirow[t]{2}{*}{ Category } & \multirow[t]{2}{*}{ GO term } & \multicolumn{4}{|c|}{ Differentially expressed predicted target genes } & \multirow{2}{*}{$\begin{array}{l}\text { Total } \\
\text { genes }\end{array}$} \\
\hline & & $\overline{D Y 2}$ & DY5 & DR2 & DR5 & \\
\hline \multirow[t]{9}{*}{ Cellular component } & extracellular region & 1 & 1 & 6 & 3 & 974 \\
\hline & cell & 35 & 33 & 85 & 85 & 24,549 \\
\hline & membrane & 18 & 17 & 29 & 36 & 9992 \\
\hline & cell junction & 0 & 1 & 2 & 2 & 385 \\
\hline & macromolecular complex & 4 & 5 & 14 & 16 & 3200 \\
\hline & organelle & 30 & 31 & 79 & 80 & 21,904 \\
\hline & organelle part & 4 & 8 & 20 & 22 & 4646 \\
\hline & membrane part & 9 & 5 & 9 & 11 & 4082 \\
\hline & cell part & 35 & 33 & 85 & 85 & 24,618 \\
\hline \multirow[t]{10}{*}{ Molecular function } & nucleic acid binding transcription factor activity & 3 & 5 & 11 & 10 & 1052 \\
\hline & catalytic activity & 20 & 12 & 39 & 40 & 17,825 \\
\hline & receptor activity & 0 & 0 & 1 & 1 & 222 \\
\hline & structural molecule activity & 0 & 1 & 3 & 6 & 753 \\
\hline & transporter activity & 3 & 2 & 3 & 2 & 1876 \\
\hline & binding & 25 & 26 & 57 & 53 & 20,072 \\
\hline & electron carrier activity & 0 & 0 & 0 & 4 & 834 \\
\hline & antioxidant activity & 0 & 1 & 0 & 1 & 370 \\
\hline & enzyme regulator activity & 1 & 0 & 1 & 0 & 344 \\
\hline & molecular transducer activity & 0 & 0 & 1 & 1 & 456 \\
\hline \multirow[t]{16}{*}{ Biological process } & reproduction & 1 & 1 & 0 & 1 & 503 \\
\hline & immune system process & 2 & 3 & 2 & 4 & 454 \\
\hline & metabolic process & 21 & 19 & 50 & 56 & 21,790 \\
\hline & cellular process & 22 & 23 & 60 & 62 & 19,163 \\
\hline & reproductive process & 3 & 3 & 7 & 9 & 1722 \\
\hline & signaling & 2 & 1 & 8 & 8 & 1423 \\
\hline & multicellular organismal process & 6 & 4 & 14 & 15 & 2556 \\
\hline & developmental process & 8 & 5 & 16 & 19 & 3341 \\
\hline & growth & 2 & 1 & 5 & 4 & 595 \\
\hline & single-organism process & 21 & 18 & 47 & 49 & 14,355 \\
\hline & rhythmic process & 0 & 1 & 0 & 0 & 59 \\
\hline & response to stimulus & 12 & 8 & 27 & 30 & 7834 \\
\hline & localization & 7 & 9 & 19 & 14 & 4192 \\
\hline & multi-organism process & 1 & 2 & 4 & 6 & 1376 \\
\hline & biological regulation & 16 & 12 & 34 & 35 & 6552 \\
\hline & cellular component organization or biogenesis & 6 & 3 & 14 & 12 & 3785 \\
\hline
\end{tabular}

DY2 and DY5 represent the differentially expressed predicted target genes at 2 and $5 \mathrm{~d}$ after YC05-179 was inoculated with Sporisorium scitamineum, whereas DR2 and DR5 represent the differentially expressed predicted target genes at 2 and $5 \mathrm{~d}$ after ROC22 was inoculated S. scitamineum. YC05-179, smut-resistant genotype; ROC22, smut-susceptible genotype

(protein Rf1, mitochondrial precursor), Sugarcane_Unigene_ BMK.59405 (nuclear transport factor 2), Sugarcane Unigene_BMK.41321 (hypothetical protein), gi34973960 (pleckstrin homology domain-containing protein 1), Sugarcane_Unigene_BMK.39426 (hypothetical protein),
Sugarcane_Unigene_BMK.2023 (uncharacterized protein LOC101772317 isoform X1) and Sugarcane_Unigene_ BMK.33875 (uncharacterized protein LOC113064467). After inoculation, 58 predicted target genes were differentially expressed only in ROC22, which were gi35049661 
Table 4 KEGG enrichment results of differentially expressed predicted target genes in YC05-179 and ROC22 inoculated with Sporisorium scitamineum for 2 and $5 \mathrm{~d}$

\begin{tabular}{|c|c|c|c|c|c|c|}
\hline Library & Kegg_pathway & ko_id & Cluter_frequency & $P$-value & Corrected_P-value & Enrichment_factor \\
\hline \multirow[t]{11}{*}{ DY2 } & Plant hormone signal transduction & ko04075 & 2 out of $9,22.22 \%$ & 0.071009 & 0.710085 & 0.22 \\
\hline & Ubiquinone and other terpenoid-quinone biosynthesis & ko00130 & 1 out of $9,11.11 \%$ & 0.071806 & 0.71806 & 0.07 \\
\hline & Glycerolipid metabolism & ko00561 & 1 out of $9,11.11 \%$ & 0.126198 & 1 & 0.13 \\
\hline & Photosynthesis & ko00195 & 1 out of $9,11.11 \%$ & 0.137578 & 1 & 0.15 \\
\hline & Arginine and proline metabolism & ko00330 & 1 out of $9,11.11 \%$ & 0.199175 & 1 & 0.22 \\
\hline & Ubiquitin mediated proteolysis & ko04120 & 1 out of $9,11.11 \%$ & 0.213623 & 1 & 0.24 \\
\hline & Plant-pathogen interaction & ko04626 & 1 out of $9,11.11 \%$ & 0.235498 & 1 & 0.26 \\
\hline & Cysteine and methionine metabolism & ko00270 & 1 out of $9,11.11 \%$ & 0.236767 & 1 & 0.27 \\
\hline & Oxidative phosphorylation & ko00190 & 1 out of $9,11.11 \%$ & 0.313078 & 1 & 0.37 \\
\hline & RNA transport & ko03013 & 1 out of $9,11.11 \%$ & 0.371941 & 1 & 0.45 \\
\hline & SNARE interactions in vesicular transport & ko04130 & 1 out of $10,10 \%$ & 0.055905 & 0.670858 & 0.06 \\
\hline \multirow[t]{11}{*}{ DY5 } & Plant hormone signal transduction & ko04075 & 2 out of $10,20 \%$ & 0.085911 & 1 & 0.25 \\
\hline & Tryptophan metabolism & ko00380 & 1 out of $10,10 \%$ & 0.089401 & 1 & 0.09 \\
\hline & Pentose and glucuronate interconversions & ko00040 & 1 out of $10,10 \%$ & 0.092691 & 1 & 0.10 \\
\hline & Glycerolipid metabolism & ko00561 & 1 out of $10,10 \%$ & 0.139209 & 1 & 0.15 \\
\hline & Photosynthesis & ko00195 & 1 out of $10,10 \%$ & 0.151658 & 1 & 0.16 \\
\hline & Fructose and mannose metabolism & ko00051 & 1 out of $10,10 \%$ & 0.154745 & 1 & 0.17 \\
\hline & Peroxisome & ko04146 & 1 out of $10,10 \%$ & 0.201316 & 1 & 0.22 \\
\hline & Ubiquitin mediated proteolysis & ko04120 & 1 out of $10,10 \%$ & 0.234362 & 1 & 0.26 \\
\hline & Plant-pathogen interaction & ko04626 & 1 out of $10,10 \%$ & 0.257991 & 1 & 0.29 \\
\hline & RNA transport & ko03013 & 1 out of $10,10 \%$ & 0.403603 & 1 & 0.50 \\
\hline & Ribosome & ko03010 & 1 out of $10,10 \%$ & 0.544618 & 1 & 0.76 \\
\hline \multirow[t]{21}{*}{ DR2 } & Plant hormone signal transduction & ko04075 & 4 out of $24,16.67 \%$ & 0.029416 & 0.705974 & 0.30 \\
\hline & Phenylalanine, tyrosine and tryptophan biosynthesis & ko00400 & 2 out of $24,8.33 \%$ & 0.029491 & 0.707793 & 0.14 \\
\hline & Photosynthesis & ko00195 & 2 out of $24,8.33 \%$ & 0.05751 & 1 & 0.20 \\
\hline & Histidine metabolism & ko00340 & 1 out of $24,4.17 \%$ & 0.117722 & 1 & 0.12 \\
\hline & SNARE interactions in vesicular transport & ko04130 & 1 out of $24,4.17 \%$ & 0.129112 & 1 & 0.14 \\
\hline & Ubiquitin mediated proteolysis & ko04120 & 2 out of $24,8.33 \%$ & 0.13054 & 1 & 0.32 \\
\hline & Cysteine and methionine metabolism & ko00270 & 2 out of $24,8.33 \%$ & 0.157229 & 1 & 0.35 \\
\hline & Fatty acid biosynthesis & ko00061 & 1 out of $24,4.17 \%$ & 0.204988 & 1 & 0.23 \\
\hline & Pentose and glucuronate interconversions & ko00040 & 1 out of $24,4.17 \%$ & 0.208438 & 1 & 0.23 \\
\hline & Porphyrin and chlorophyll metabolism & ko00860 & 1 out of $24,4.17 \%$ & 0.222097 & 1 & 0.25 \\
\hline & Ascorbate and aldarate metabolism & ko00053 & 1 out of $24,4.17 \%$ & 0.232192 & 1 & 0.26 \\
\hline & Oxidative phosphorylation & ko00190 & 2 out of $24,8.33 \%$ & 0.256669 & 1 & 0.49 \\
\hline & Glycine, serine and threonine metabolism & ko00260 & 1 out of $24,4.17 \%$ & 0.283948 & 1 & 0.33 \\
\hline & Pentose phosphate pathway & ko00030 & 1 out of $24,4.17 \%$ & 0.290177 & 1 & 0.34 \\
\hline & Fructose and mannose metabolism & ko00051 & 1 out of $24,4.17 \%$ & 0.332351 & 1 & 0.40 \\
\hline & Arginine and proline metabolism & ko00330 & 1 out of $24,4.17 \%$ & 0.447393 & 1 & 0.58 \\
\hline & Phagosome & ko04145 & 1 out of $24,4.17 \%$ & 0.454667 & 1 & 0.60 \\
\hline & Ribosome biogenesis in eukaryotes & ko03008 & 1 out of $24,4.17 \%$ & 0.478256 & 1 & 0.64 \\
\hline & Amino sugar and nucleotide sugar metabolism & ko00520 & 1 out of $24,4.17 \%$ & 0.478256 & 1 & 0.64 \\
\hline & Carbon fixation in photosynthetic organisms & ko00710 & 1 out of $24,4.17 \%$ & 0.507463 & 1 & 0.70 \\
\hline & Starch and sucrose metabolism & ko00500 & 1 out of $24,4.17 \%$ & 0.513977 & 1 & 0.71 \\
\hline
\end{tabular}


Table 4 KEGG enrichment results of differentially expressed predicted target genes in YC05-179 and ROC22 inoculated with Sporisorium scitamineum for 2 and $5 \mathrm{~d}$ (Continued)

\begin{tabular}{|c|c|c|c|c|c|c|}
\hline Library & Kegg_pathway & ko_id & Cluter_frequency & $P$-value & Corrected_P-value & Enrichment_factor \\
\hline & Ribosome & ko03010 & 2 out of $24,8.33 \%$ & 0.551296 & 1 & 0.91 \\
\hline & Glycolysis / Gluconeogenesis & ko00010 & 1 out of $24,4.17 \%$ & 0.636432 & 1 & 0.99 \\
\hline & Protein processing in endoplasmic reticulum & ko04141 & 1 out of $24,4.17 \%$ & 0.67661 & 1 & 1.10 \\
\hline \multirow[t]{20}{*}{ DR5 } & Photosynthesis & ko00195 & 3 out of $24,12.5 \%$ & 0.006618 & 0.132362 & 0.13 \\
\hline & Plant hormone signal transduction & ko04075 & 4 out of $24,16.67 \%$ & 0.029416 & 0.588312 & 0.30 \\
\hline & Ribosome & ko03010 & 4 out of $24,16.67 \%$ & 0.10279 & 1 & 0.45 \\
\hline & Selenocompound metabolism & ko00450 & 1 out of $24,4.17 \%$ & 0.106189 & 1 & 0.11 \\
\hline & Histidine metabolism & ko00340 & 1 out of $24,4.17 \%$ & 0.117722 & 1 & 0.12 \\
\hline & Ubiquitin mediated proteolysis & ko04120 & 2 out of $24,8.33 \%$ & 0.13054 & 1 & 0.32 \\
\hline & Cysteine and methionine metabolism & ko00270 & 2 out of $24,8.33 \%$ & 0.157229 & 1 & 0.35 \\
\hline & 6--Sulfur metabolism & ko00920 & 1 out of $24,4.17 \%$ & 0.169683 & 1 & 0.18 \\
\hline & Ubiquinone and other terpenoid-quinone biosynthesis & ko00130 & 1 out of $24,4.17 \%$ & 0.180429 & 1 & 0.20 \\
\hline & Tryptophan metabolism & ko00380 & 1 out of $24,4.17 \%$ & 0.201523 & 1 & 0.22 \\
\hline & Pentose and glucuronate interconversions & ko00040 & 1 out of $24,4.17 \%$ & 0.208438 & 1 & 0.23 \\
\hline & Phenylalanine, tyrosine and tryptophan biosynthesis & ko00400 & 1 out of $24,4.17 \%$ & 0.238852 & 1 & 0.27 \\
\hline & Oxidative phosphorylation & ko00190 & 2 out of $24,8.33 \%$ & 0.256669 & 1 & 0.49 \\
\hline & Glycine, serine and threonine metabolism & ko00260 & 1 out of $24,4.17 \%$ & 0.283948 & 1 & 0.33 \\
\hline & Fructose and mannose metabolism & ko00051 & 1 out of $24,4.17 \%$ & 0.332351 & 1 & 0.40 \\
\hline & Peroxisome & ko04146 & 1 out of $24,4.17 \%$ & 0.417358 & 1 & 0.53 \\
\hline & Arginine and proline metabolism & ko00330 & 1 out of $24,4.17 \%$ & 0.447393 & 1 & 0.58 \\
\hline & Phagosome & ko04145 & 1 out of $24,4.17 \%$ & 0.454667 & 1 & 0.60 \\
\hline & Purine metabolism & ko00230 & 1 out of $24,4.17 \%$ & 0.644512 & 1 & 1.01 \\
\hline & Protein processing in endoplasmic reticulum & ko04141 & 1 out of $24,4.17 \%$ & 0.67661 & 1 & 1.10 \\
\hline
\end{tabular}

DY2 and DY5 represent the differentially expressed predicted target genes at $2 \mathrm{~d}$ and $5 \mathrm{~d}$ after YC05-179 was inoculated with S. scitamineum, whereas DR2 and DR5 represent the differentially expressed predicted target genes at $2 \mathrm{~d}$ and $5 \mathrm{~d}$ after ROC22 was inoculated S. scitamineum. YC05-179, smut-resistant genotype: ROC22, smut-susceptible genotype

(SERK2), gi35329294 (auxin response factor 14, ARF14), Sugarcane_Unigene_BMK.71301 (ARF17), gi35090530 (ubiquitin-conjugating enzyme E2 5, UBC5), Sugarcane Unigene_BMK.54856 (S-adenosylmethionine synthase 1), Sugarcane_Unigene_BMK.64656 (EIL3), and Sugarcane_ Unigene_BMK.53115 (oxygen-evolving enhancer protein 2 ), etc.. These genes could be used as candidates for the further smut-resistance research.

\section{YC05-179 continuously, ROC22 non-continuously} differentially expressed predicted target genes

A comparative analysis of continuously differentially expressed predicted target genes in YC05-179 and non-continuously differentially expressed predicted target genes in ROC22 was performed to obtain predicted target genes with transient response or lagged response to S. scitamineum in ROC22 (Fig. 5). Among these, seven predicted target genes Sugarcane_Unigene_BMK.3158 (formin-like protein 16), Sugarcane_Unigene_BMK.49383 (tRNA-splicing endonuclease subunit Sen2), Sugarcane_Unigene_BMK.56064 (heterogeneous nuclear ribonucleoprotein R-like), Sugarcane_Unigene_BMK.57076 (hypothetical protein), Sugarcane_Unigene_BMK.51607 (Zinc finger MYM-type protein), gi34944423 (Sn1-specific diacylglycerol lipase beta), and Sugarcane_Unigene_BMK.45288 (serine/arginine repetitive matrix protein 2) were shared between continuously differentially expressed predicted target genes in YC05179 and non-continuously differentially expressed predicted target genes in DR2. Sugarcane_Unigene_BMK.51989 (hypersensitive-induced response protein 1, HIR1), gi36030075 (lysine histidine transporter 1, LHT1), Sugarcane_Unigene_BMK.70126 (E3 ubiquitin-protein ligase SDIR1), and Sugarcane_Unigene_BMK.62668 (MLO-like protein 13) were shared between continuously differentially expressed predicted target genes in YC05-179 and non-continuously differentially expressed predicted target genes in DR5. 


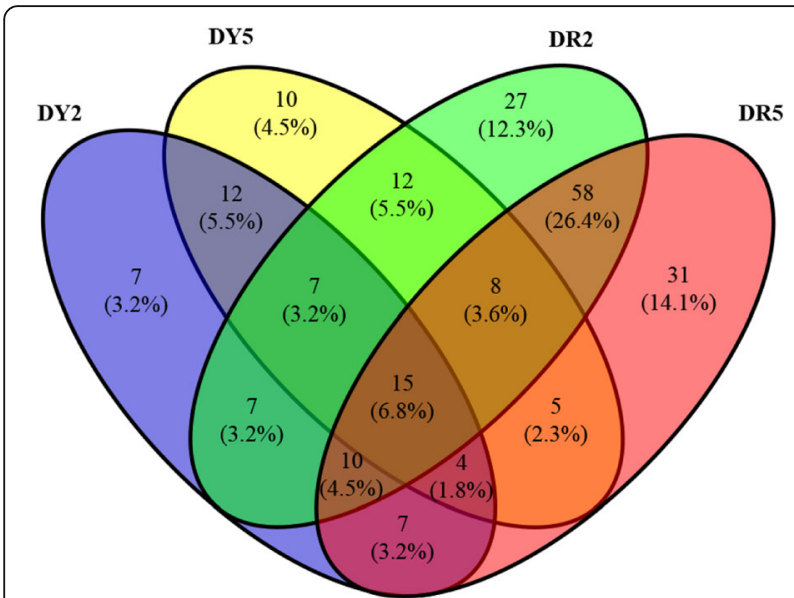

Fig. 5 Venn diagram of differentially expressed predicted target genes in YC05-179 (DY) and ROC22 (DR) inoculated with Sporisorium scitamineum for $2 \mathrm{~d}$ and $5 \mathrm{~d}$. The number and percentage was the quantity and proportion of differentially expressed predicted target genes in library specific or common, respectively. YC05-179, smut-resistant genotype; ROC22, smut-susceptible genotype

\section{Non-continuously differentially expressed predicted target genes specific to YC05-179}

Figure 5 showed that in YC05-179, in addition to 12 species-specific continuously differentially expressed predicted target genes, there were seven predicted target genes that were differentially expressed only at $2 \mathrm{~d}$ after inoculation, including Sugarcane_Unigene_BMK.64437 (NAC domain-containing protein 21/22), and gi35098237 (Myb-related protein), etc.. In addition, 10 predicted target genes were differentially expressed only at $5 \mathrm{~d}$ after inoculation, including Sugarcane_Unigene_BMK.71328 (40S ribosomal protein), Sugarcane_Unigene_BMK.75459 (proline-rich receptor-like protein kinase, PERK2), and Sugarcane_Unigene_BMK.44399 (phosphate carrier protein), etc.. As for those genes differentially expressed only in the resistant variety, they could be considered as candidate smut-resistance genes that require further studies.

\section{qRT-PCR validation of the expression level of miRNAs and} their corresponding predicted target genes

qRT-PCR analysis of miRNAs and their corresponding target genes will not only verify the accuracy of our degradome sequencing results, but also determine the miRNA-mediated regulatory role of miRNAs and their predicted target genes in sugarcane responses to $S$. scitamineum stress. The results showed that the expression level of 13 predicted target genes in qRT-PCR analysis and degradome sequencing was similar, but not completely consistent (Fig. 6). There was also a certain deviation in the differences of gene expression fold. The predicted target genes and their corresponding miRNAs were expressed in opposite patterns in at least one sugarcane variety. However, the expression patterns of different predicted target genes and their corresponding miRNAs in two sugarcane varieties (YC05-179 and ROC22) varied as follows:

(i) Ubiquitin carboxyl-terminal hydrolase isozyme L5-like (UCH-L5), protein argonaute $1 \mathrm{~B}(A G O 1 B)$, and ARF8 followed a negative miRNA-mediated regulatory mode in two sugarcane varieties. The expression patterns of predicted target genes $U C H-L 5, A G O 1 B$, and ARF8 were opposite to that of their corresponding miRNAs, i.e., $U C H-L 5, A G O 1 B$, and $A R F 8$ (slightly decreased but not significant) were downregulated, whereas the corresponding miR529-3p, miR168a-5p, and miR160a were upregulated in YC05-179, and the expression pattern in ROC22 was the opposite (Fig. 6A).

(ii) Auxin-induced protein $(A I P)$, cinnamoyl-CoA reductase $(C C R)$, and $S$-adenosylmethionine decarboxylase $(S A M D C)$ fit the negative miRNA-mediated regulatory mode only in ROC22. While the expression trends of predicted target genes and their corresponding miRNA in YC05-179 were consistent, i.e., $A I P, C C R$, and $S A M D C$ were upregulated, the corresponding miR5293, nov-mir-132, and miR162a were downregulated in ROC22, whereas these predicted target genes and their corresponding miRNAs were upregulated in YC05-179 (Fig. 6B).

(iii) EIL3 and HIR1 were upregulated and followed the negative miRNA-mediated regulatory mode only in ROC22. Whereas the expression level of EIL3 and HIR1 in YC05-179 showed little change, the expression amount of their corresponding nov-mir-143 and miR5368 varied greatly and all was upregulated (Fig. 6C).

(iv) Growth-regulating factor 8 (GRF8), glycerol kinase $(G K), P P 2 C$, mildew resistance locus o $(M L O)$, and Myb-related protein Hv33 (MYB2) fit the negative miRNA-mediated regulatory mode only in YC05-179 (Fig. 6D). GRF8 was downregulated in both YC05-179 and ROC22, and the corresponding nov-mir-18, nov-mir-66, and miR396e-5p were all upregulated in YC05-179, but upregulated in ROC22 only at $5 \mathrm{~d}$. GK was upregulated at $2 \mathrm{~d}$ and downregulated at $5 \mathrm{~d}$ after inoculation with $S$. scitamineum in YC05-179, whereas the corresponding nov-mir-63 showed the opposite pattern. The expression level of $G K$ in ROC22 decreased with prolongation of inoculation time. The expression pattern of nov-mir-63 was the same as that in YC05-179. PP2C was downregulated and the corresponding nov-mir-97 was upregulated in YC05-179 at $2 \mathrm{~d}$, but both of them were stable in ROC22. MLO was downregulated in YC05-179 and ROC22 at $2 \mathrm{~d}$, and both of them recovered at 


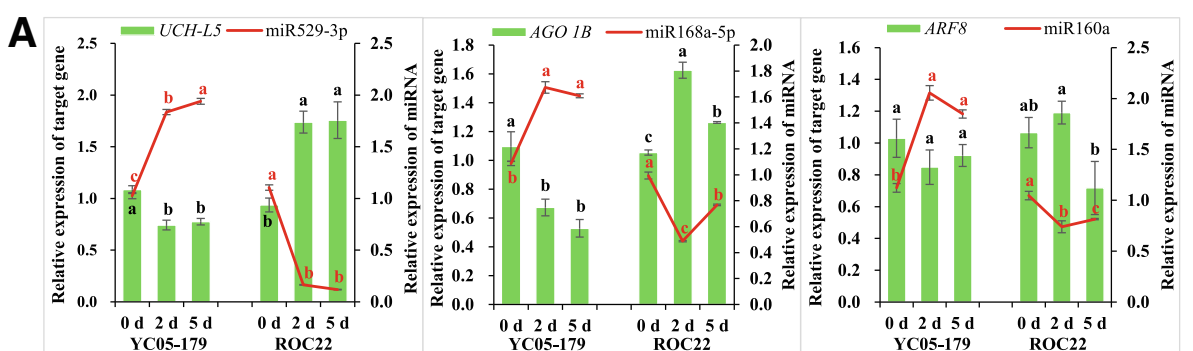

B

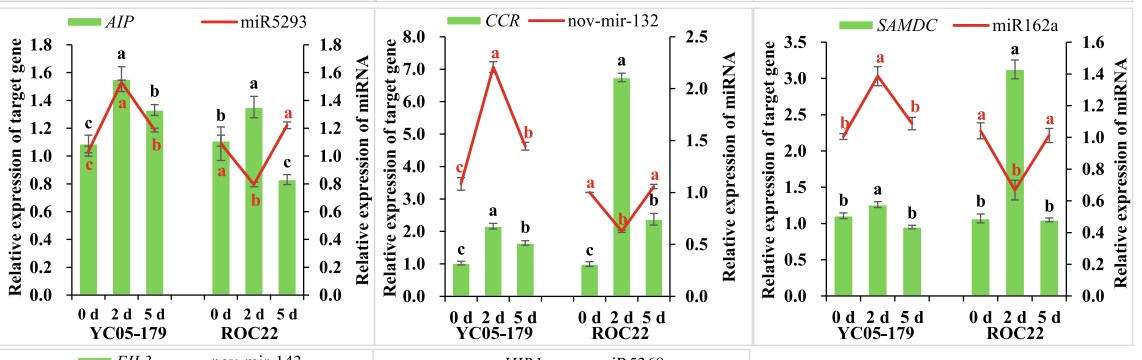

C

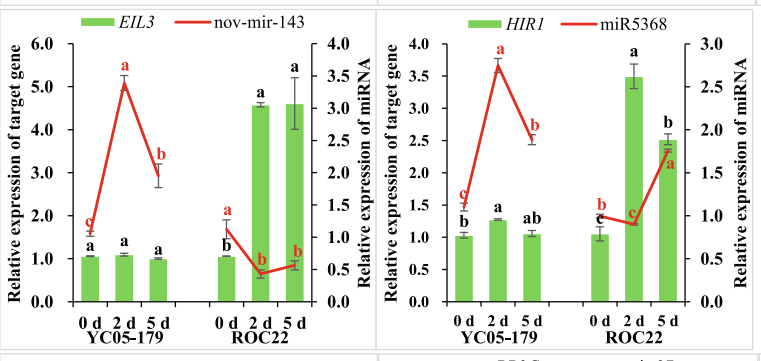

D
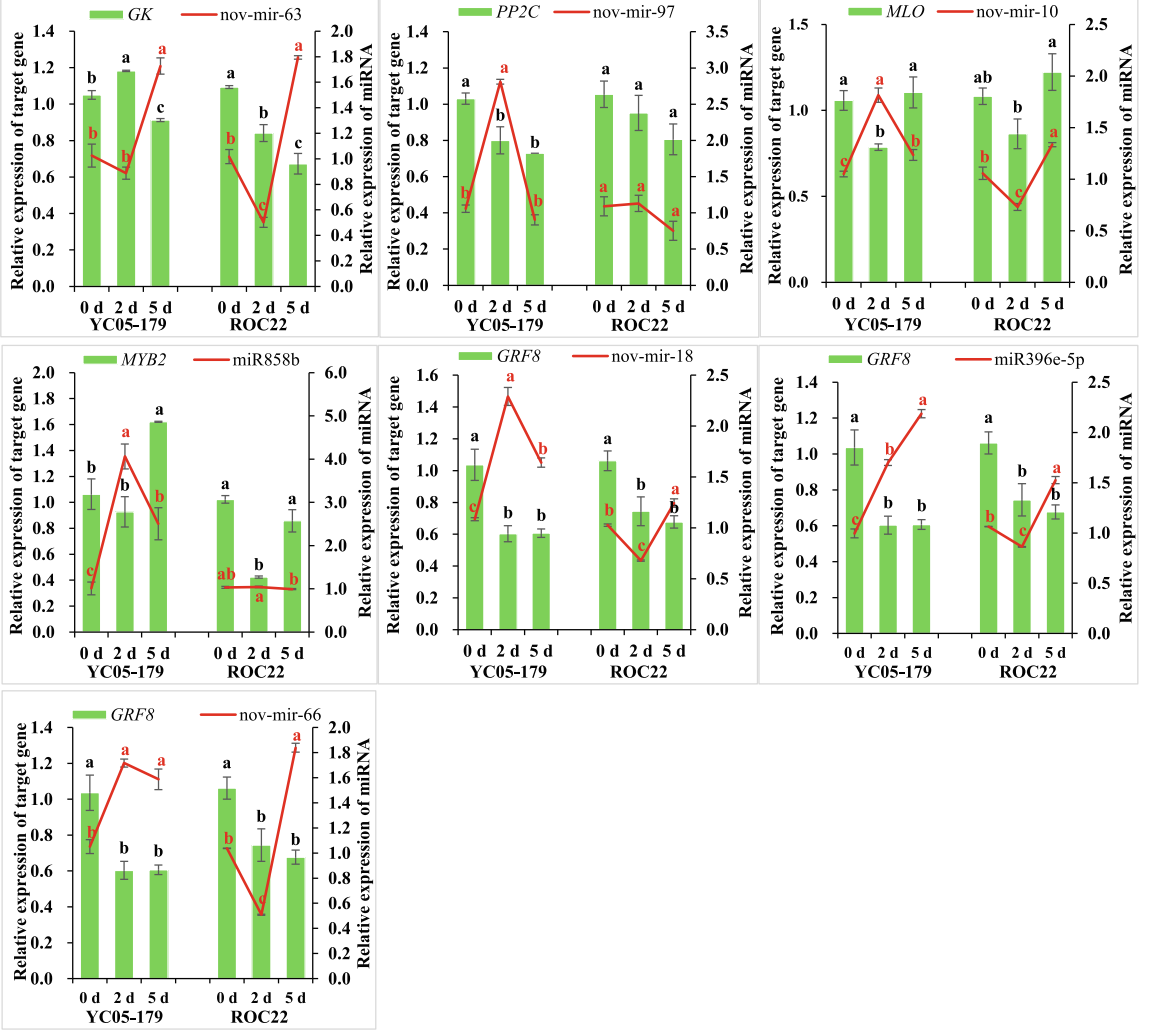

Fig. 6 (See legend on next page.) 
(See figure on previous page.)

Fig. 6 qRT-PCR validation of the expression level of predicted target genes and corresponding miRNAs under Sporisorium scitamineum stress at different process times. a Expression analysis of miR529-3p, miR168a-5p, miR160a, and their predicted target genes UCH-L5, AGO 1B, and ARF8. b Expression analysis of miR5293, nov-mir-132, miR162a, and their predicted target genes AIP, CCR, and SAMDC. c Expression analysis of nov-mir-143, miR5368, and their predicted target genes ElL3 and HIR1. d Expression analysis of nov-mir-63, nov-mir-97, nov-mir-10, miR858b, nov-mir-18, miR396e-5p, nov-mir-66, and their predicted target genes GK, PP2C, MLO, MYB2, and GRF8. All data points were means \pm standard error $(n=3)$. Different lowercase letters indicated a significant difference, as determined by the Duncan's new multiple range test $(p<0.05)$. YC05-179, smut-resistant genotype; ROC22, smut-susceptible genotype. UCH-L5, ubiquitin carboxyl-terminal hydrolase isozyme L5-like; $A G O$ 1B, protein argonaute 1B; ARF8, auxin response factor 8; AIP, auxin-induced protein; CCR, cinnamoyl-CoA reductase; SAMDC, S-adenosylmethionine decarboxylase; ElL3, ethylene-insensitive 3-like 3 protein; HIR1, hypersensitive-induced response protein 1; GK, glycerol kinase; PP2C, protein phosphatase 2C; MLO, MLO-like protein; MYB2, Myb-related protein Hv33; GRF8, growth-regulating factor 8

$5 \mathrm{~d}$. Its corresponding nov-mir-10 was significantly upregulated in YC05-179 at $2 \mathrm{~d}$ and then declined again at $5 \mathrm{~d}$, whereas the expression pattern in ROC22 was the opposite. The expression level of MYB2 in YC05-179 was higher than that in ROC22. When the transcript of $M Y B 2$ in YC05-179 was upregulated at $5 \mathrm{~d}$, it remained stable in ROC22, and the corresponding miR858b was upregulated in YC05-179 and peaked at $2 \mathrm{~d}$ but remained unchanged in ROC22.

The above results indicated the complexity of miRNA regulation of $S$. scitamineum that infects sugarcane. There was no obvious negative linear regulation between the miRNAs and their predicted target genes, and there were also significant differences in the regulation of expression patterns between different genotypes of sugarcane. The expression level of AGO 1B,UCH-L5, AIP, $C C R, E I L 3, H I R 1$, and SAMDC significantly changed at $2 \mathrm{~d}$ after inoculation, indicating that they responded earlier to S. scitamineum infection. ARF and GRF are associated with plant growth and development [59-61]. The significantly decreased expression level of GRF8 and the slightly decreased expression level of $A R F 8$ after inoculation suggests that the infection of S. scitamineum might inhibit the growth of sugarcane to a certain extent. MLO is a calcium-binding protein whose expression is negatively correlated with plant disease resistance $[62,63]$. The $M L O$ gene in sugarcane was downregulated at $2 \mathrm{~d}$ after inoculation with $S$. scitamineum compared to ROC22, with a significant decrease in expression level in YC05-179, suggesting that $M L O$ responded earlier to smut pathogen infection and that the intensity of the response was greater in the resistant variety than the susceptible one. $M Y B$ plays a more important role in the defense response of plants to stress [64]. MYB2 was upregulated in YC05-179 at $5 \mathrm{~d}$ after inoculation with $S$. scitamineum, indicating a delay in response. $G K$ is a rate-limiting enzyme in the metabolic pathway of glycerol, and its expression level is closely related to the innate immune response in plants $[65,66]$. The expression level of $G K$ in the resistant variety YC05-179 was increased and higher than that in the susceptible variety ROC22 after infection with $S$. scitamineum, which may corroborate the positive correlation between the expression level of $G K$ and smut resistance in sugarcane varieties.

\section{Discussion}

Plant miRNAs mainly regulate target gene expression by mediating target mRNAs cleavage or repressing gene translation during plant development [26, 27]. Degradome sequencing is a high-throughput method to identify miRNAs and their predicted target genes at a certain developmental stage or under specific stress in plants, which in turn may reveal the target genes of miRNAs that are related to plant development or response to stress $[28,29]$. This technique has now been successfully applied to various plants such as Populus tomentosa [57], cotton [58], rice [67], and peanut [31]. In the present study, the expression of the predicted target genes regulated by miRNA-mediated cleavage in smut-resistant and -susceptible varieties of sugarcane under the infection of $S$. scitamineum was analyzed using degradome sequencing. The results showed that an initial data amount of 122.33 $\mathrm{M}$ was obtained on six sugarcane samples. The data amount of each sample was not less than $10 \mathrm{M}$ and the Q30 was > 93\%, suggesting that the sequencing quality was relatively high. In addition, the sequence of degraded fragments obtained by degradome sequencing was the same as that of transcriptome sequencing [11], which demonstrates the accuracy of degradome sequencing results.

Degradome sequencing allows the rapid acquisition of miRNA-mediated 3' cleavage fragments containing $5^{\prime}$ monophosphate groups, and then the identification of target gene degradation sites through depth statistics and comparative analysis on the cleaved fragment $[28,29]$. In this study, a total of 309 predicted target mRNAs were detected in six libraries, corresponding to 97 known miRNAs and 112 new miRNAs, as well as 337 degradation sites. Previous studies have shown target mRNAs can be cleaved by miRNAs at multiple degradation sites, resulting in a higher number of degradation sites than target genes [56]. After S. scitamineum infection, the negative regulatory role in quantitative expression between partial selected miRNAs and their predicted target genes in 
two sugarcane genotypes was not extremely high (Fig. 6). It is possible that these potential target genes were regulated by more than one miRNAs at the translational level [68-70].

The target genes were annotated with GO, KEGG, NR, NT, Swiss-Prot and COG databases to obtain their basic information, functional classification, and involved metabolic pathways [71, 72]. In this study, predicted target genes involved in various regulatory processes of life activity such as signal transduction, ion transport, translation and posttranslational modification, energy production and transduction, and metabolism of glycerides. In addition, a miRNA precursor, namely, Sugarcane_Unigene_BMK.40037 (miR171e-3 precursor miRNA), cleaved by nov-mir-219, was found in the YC05-179 at $2 \mathrm{~d}$ after inoculation with $S$. scitamineum. The interaction process between sugarcane and $S$. scitamineum is regulated by a multi-gene network system. Correspondingly, the process of sugarcane in response to $S$. scitamineum infection is involved in the regulation of multiple metabolic pathways [73, 74]. The differentially expressed predicted target genes in YC05-179 and ROC22 were basically the same in the classification of targets, mainly playing a catalytic and binding role in response to stimulation, signaling pathway and immune process. KEGG analysis showed that the differentially expressed predicted target genes involved in plant hormone signal transduction, plant-pathogen interactions, oxidative phosphorylation, and other disease-related metabolic pathways, in which plant-pathogen interaction pathways appeared only in the differentially expressed predicted target genes of YC05-179, namely Sugarcane_Unigene_BMK.75694. Sugarcane_Unigene_ BMK.75694 is cleaved by nov-mir-63, encodes glycerol kinase, which is involved in energy production and transduction. After inoculating with S. scitamineum, the expression of Sugarcane_Unigene_BMK.75694 in YC05-179 was increased with the prolongation of inoculation time, which was opposite to that in ROC22. Sugarcane_Unigene_BMK.62557 (catalase), which participated in the peroxisomal pathway and was regulated by miR858b and nov-mir-88, was only differentially expressed in YC05-179 and ROC22 that were inoculated for $5 \mathrm{~d}$. The expression level of Sugarcane_Unigene_BMK.62557 in YC05-179 was increased with the elongation of inoculation, but was opposite to that in ROC22. This does not agree with our finding that catalase activity was higher at 2 d compared to that at $5 \mathrm{~d}$ after inoculation (Fig. 2), which may be because the expression level of other catalase family members had greater changes than that of Sugarcane_Unigene_BMK.62557, thus fitting the trend of changes in the activity of catalase. In addition, YC05-179 and ROC22 also responded to S. scitamineum infection through their own differential expression of genes. Therefore, link the changes in transcript levels of predicted target genes to observe biology of infection will provide a better insight on sugarcane in response to smut pathogen attack.

Pathogen invasion triggers various plant immune responses $[75,76]$. When infected by pathogens, the responses of plant genes may differ such as early or late stress responses or high or low expression level [11]. miRNAs mainly regulate target gene expression by cleavage, which in turn influences the life processes of plants $[26,27]$. At the same time, the response of plants to pathogen infection involves multiple metabolic pathways and several genes $[10,13]$. This study showed that inoculation with S. scitamineum induces changes in multiple resistance-related metabolic pathways in YC05-179 and ROC22 as following:

\section{Lignin biosynthesis pathway}

Lignin biosynthesis is a branch of the phenylpropanoid metabolic pathway that plays an important role in plant disease resistance [77]. CCR is a key enzyme that catalyzes lignin biosynthesis and promotes the formation of lignin $[78,79]$. CCR first catalyzes the formation of corresponding aldehydes by coumaryl-CoA, feruloyl-CoA and sinapoyl-CoA, which are then catalyzed by cinnamyl alcohol dehydrogenase (CAD) to form lignin monomers $[78,79]$. Previous studies have found that the reduced expression of $C C R$ can cause a significant decrease in lignin content [80]. In addition, CCR gene expression in plants is upregulated and the accumulation of lignin monomer is increased during fungal infections [81, 82]. PPO has often been suggested to participate in plant defense against pathogens and pests by promoting the formation of lignin and quinones [56, 83]. Li and Steffens reported that overexpression of $P P O$ in tomato plants results in enhanced resistance to Pseudomonas syringae pv. tomato [56]. In this study, degradome sequencing analysis showed that nov-mir-132 targets the CCR gene and regulates its expression by cleavage. After inoculation with S. scitamineum, the expression level of $C C R$ in YC05-179 and ROC22 was upregulated (Fig. 6B), which coincided with the increase in PPO activity at 2 and $5 \mathrm{~d}$ after inoculation (Fig. 2), suggesting that high expression of CCR transcript and PPO activity may promote the synthesis and accumulation of lignin to actively respond to $S$. scitamineum infection.

\section{Ubiquitin-mediated pathway of protein degradation}

Ubiquitination is a common protein modification in eukaryotes [84]. Ubiquitin-mediated protein degradation pathways such as jasmonic acid (JA) [85], salicylic acid (SA) [86], and effector-triggered immunity (ETI) [87] are involved in plant immune responses. Deubiquitination enzyme (DUB) has been shown to efficiently cleave ubiquitin-labeled target proteins and regulate ubiquitin-mediated 
metabolic pathways [88]. UCH-L5, a deubiquitinating enzyme belonging to the ubiquitin carboxy terminal hydrolase family, is closely related to the ubiquitin degradation pathway [89]. In this study, miR529-3p targets UCH-L5, was downregulated in YC05-179 after inoculation with $S$. scitamineum, whereas upregulated in ROC22 (Fig. 6A), suggesting that $U C H-L 5$ may undergo ubiquitinmediated protein degradation pathways to respond to $S$. scitamineum infection.

\section{Interaction pathways between plants and pathogens}

Interaction between plants and pathogens will stimulate various defense mechanisms in plants such as the formation and accumulation of phytoalexins and disease-related proteins, second messenger production, hypersensitive reactions (HRs), and programmed cell death (PCD). GK catalyzes the phosphorylation of glycerol, which is a key rate-limiting enzyme in the glycerol metabolic pathway [90]. Nonhost resistance 1 (NHO1), a member of GK, is involved in the JA and SA signal transduction pathways and plays a role in plant disease resistance $[66,91]$. The $\mathrm{NHO}$ gene is necessary for the R-gene related pathway and its expression can be activated by flagellin [65]. After inoculation with Xanthomonas oryzae, the expression level of OsNHO1 in rice rapidly increased within $3 \mathrm{~h}$, peaked at $9 \mathrm{~h}$, then gradually decreased and dropped to that of the control at $1 \mathrm{~d}$, indicating that NHO1 is highly responsive to $X$. oryzae in rice [92]. In this study, nov-mir-63 targets GK. KEGG pathway analysis showed that this GK has NHO1 activity and participates in the plant-pathogen interaction pathway and glycerolipid metabolism. Moreover, after inoculation with S. scitamineum, the $G K$ expression level was upregulated in the resistant variety YC05-179 but downregulated in the susceptible variety $\mathrm{ROC} 22$ at $2 \mathrm{~d}$ (Fig. $6 \mathrm{D}$ ), suggesting that the $G K$ gene may play a positive role in sugarcane resistance to $S$. scitamineum.

$\mathrm{Ca}^{2+}$ is a second messenger involved in plant-pathogen interactions. $M L O$ is a recessive susceptible gene. $\mathrm{Ca}^{2+}$-mediated $\mathrm{MLO}$ binds to calmodulin and is negatively correlation to plant disease resistance [62]. Plants often show susceptibility when the $M L O$ gene normally expresses the MLO protein, whereas exhibit broad-spectrum disease resistance when the $M L O$ gene does not express or express non-functional proteins [63]. Previous studies have shown that mol mutations can improve plant resistance to bacteria and fungi [93, 94]. Therefore, in plants, the $M L O$ gene is equivalent to a susceptible gene. The $M L O$ gene has been associated with resistance to powdery mildew of wheat [95], as well as resistance to leaf blight of barley [96], necrosis of infected parts, and suppression of pathogen expansion [96]. In this study, we found that nov-mir-10 could target $M L O$. After inoculation with $S$. scitamineum, $M L O$ was downregulated in $\mathrm{YC} 05-179$ at $2 \mathrm{~d}$, and its expression level was lower than that in ROC22 (Fig. 6D). It has been suggested that nov-mir-10 in YC05-179 could more efficiently cleave $M L O$, thereby reducing the inhibition of defense response by the MLO protein and improving sugarcane resistance to smut pathogen.

\section{Phytohormone signal transduction pathway}

Phytohormones play a role in plant growth and development and responses to environmental stresses [97]. Phytohormones mainly include auxin, gibberellin acid (GA), cytokinins (CKs), ethylene (ETH), SA, JA, polyamines, and brassinosteroids (BRs). Among these phytohormones, SA, JA, ETH, and BR are disease resistance signaling molecules, whereas ETH and auxin biosynthesis promote each other and participate in plant responses to stress [97]. Ethylene insensitive 3 (EIN3) and EIN3-like 1 (EIL1) are transcription factors in the ethylene signaling pathway that promote the expression of ethylene response factor 1 (ERF1) and thereby regulate defense genes in response to pathogen infection [98]. In our previous proteomics research on sugarcane at $2 \mathrm{~d}$ after inoculation with $S$. scitamineum by iTRAQ analysis, four proteins involved in the ETH pathway were observed, including two 1aminocyclopropane-1-carboxylate oxidases (ACOs) that were responsible for ETH biosynthesis, as well as one EIN3 and one ERF1 that was responsible for ETH signaling [12]. One ACO (gi35014290) was upregulated in both sugarcane genotypes (YC05-179 and ROC22) and the other one (gi41615358) was downregulated in ROC22 only, but remained unchanged in YC05-179. One EIN3 (Sugarcane_Unigene_BMK.65773) and one ERF1 (gi35045219) were both upregulated in YC05179 , whereas it remained stable in ROC22 [12]. In this study, another EIL3 gene (Sugarcane_Unigene_BMK.64656) was targeted by nov-mir-143. After inoculation with $S$. scitamineum, the expression of EIL3 (Sugarcane_Unigene_BMK.64656) in YC05-179 was stable, but in ROC22 it was rapidly increased at $2 \mathrm{~d}$ and $5 \mathrm{~d}$ (Fig. 6C). It is speculated that smut pathogen attack enhances sugarcane ethylene metabolism, which is involved in EIL3 in ROC22 and in turn favors resistance to S. scitamineum infection [99]. However, YC05-179 responded to smut pathogen infection by other family genes in the ETH pathway. In addition, we also identified an AIP gene. KEGG analysis showed that AIP has small auxin up RNAs (SAUR) activity. SAUR plays a negative regulatory role in auxin biosynthesis and transport [100]. After inoculation with S. scitamineum, the expression level of AIP was upregulated in YC05-179 at 2 and $5 \mathrm{~d}$ and increased at $2 \mathrm{~d}$ in ROC22 by qRT-PCR (Fig. 6B). However, since this result differs from the AIP expression patterns obtained from degradome sequencing, further experiments are needed to verify how $A I P$ responds to the infection by $S$. scitamineum. SAMDC and 
S-adenosylmethionine synthetase (SAMS) are important genes for polyamine biosynthesis [101, 102]. Previous studies have shown that overexpression of the SAMDC gene can increase wilt resistance that is induced by Verticillium dahliae and Fusarium oxysporum in transgenic tobacco plants [103]. The expression of a SAMS gene in sugarcane increases after infection with $S$. scitamineum [104]. We found that miR162a targets $S A M D C$ after inoculation with $S$. scitamineum and promotes the expression of SAMDC in both YC05-179 and ROC22 at $2 \mathrm{~d}$ (Fig. 6B), indicating that infection by $S$. scitamineum may enhance polyamine metabolism pathway to improve sugarcane smut resistance at the early stage.

\section{Resistance-related transcription factors}

Infection by pathogenic bacteria can stimulate plant transcription factors to participate in defensive responses. MYB is a typical transcription factor in plants that regulates phenylpropanoid metabolism [105] and is involved in hormonal signal transduction pathways such as auxin [106], JA [107], and ETH [107]. MYB is also involved in the systematic acquired resistance (SAR) and HR reactions [108]. Previous studies have found that MYB regulates PAL synthesis and is a positive regulator of phenylpropanoid anabolism [105]. In addition, MYB can be induced by exogenous stresses such as JA and SA, or TMV infection, and it can also activate disease-resistant defense responses involving the $P R$ gene [109]. In this study, we found that MYB2 could be targeted by miR858b. After $5 \mathrm{~d}$ of inoculation with S. scitamineum, MYB2 gene was upregulated in YC05-179 and remained stable in ROC22 (Fig. 6D), which coincided with the expression pattern of PPO activity (Fig. 2), suggesting that miR858b may have different cleavage effects on MYB2 then regulate PAL synthesis in different resistant varieties of sugarcane, ultimately leading to different levels of resistance to smut in YC05-179 and ROC22. Similarly, Yang et al. demonstrated that a novel anther-specific myb gene (NtMYBAS1) from tobacco was a functional anther-specific transcription factor, which was likely to be a positive regulator of PAL synthesis in sporophytic [105].

\section{miRNA feedback regulation}

Previous studies have found that miRNAs can regulate target gene expression and involved in plant growth, development, and stress response, but also feedback regulate their metabolic synthesis [27]. Xie et al. found that Arabidopsis dicer-like1 (DCL1), which plays an important role in the formation of mature miRNAs, is subject to negative feedback regulation through the activity of miR162 [110]. AGO protein is an important part of RNA-induced silencing complex (RISC) with the function of cleaving miRNA target gene or inhibiting translation [111]. miR168 can target AGO protein and thus regulate the miRNA- regulated target genes in plants through changes in AGO protein expression [111]. In this study, $A G O 1 B$ was targeted by miR168a-5p. After inoculation with $S$. scitamineum, the upregulated expression of miR168a-5p in YC05179 causes a decrease in the expression level of target $A G O$ $1 B$ and may promote miRNA-mediated accumulation of disease-related target genes, which in turn resists further infection of S. scitamineum. Meanwhile, after inoculation with S. scitamineum, the expression patterns of $A G O 1 B$ and miR168a-5p in ROC22 were opposite to that in YC05179 , i.e., miR168a-5p was downregulated and the expression level of $A G O 1 B$ was upregulated, suggesting that the miRNA self-feedback pathway may involve in sugarcane responses to S. scitamineum.

\section{Conclusions}

In the present study, the S. scitamineum was rapidly proliferated and the enzyme activities of POD, SOD, CAT, PPO, PAL, and TAL in the reactive oxygen species metabolic pathway and phenylpropanoid metabolic pathway were significantly changed at 2 and $5 \mathrm{~d}$ in the compatible and incompatible interactions between sugarcane and S. scitamineum. Furthermore, 97 known miRNAs and 112 novel miRNAs with 309 predicted target genes were identified by degradome sequencing. GO and KEGG pathway analyses showed that many predicted target genes enriched in regulation and metabolism. qRT-PCR validation demonstrated that there was no obvious negative regulatory relationship between miRNAs and their target genes. This study elucidates the underlying response of sugarcane to S. scitamineum infection and provides useful information on the interplay between miRNAs and their predicted targets. In the future, genetic transformations can be done to further our understanding on whether these genes enhance smut resistance in sugarcane.

\section{Additional files}

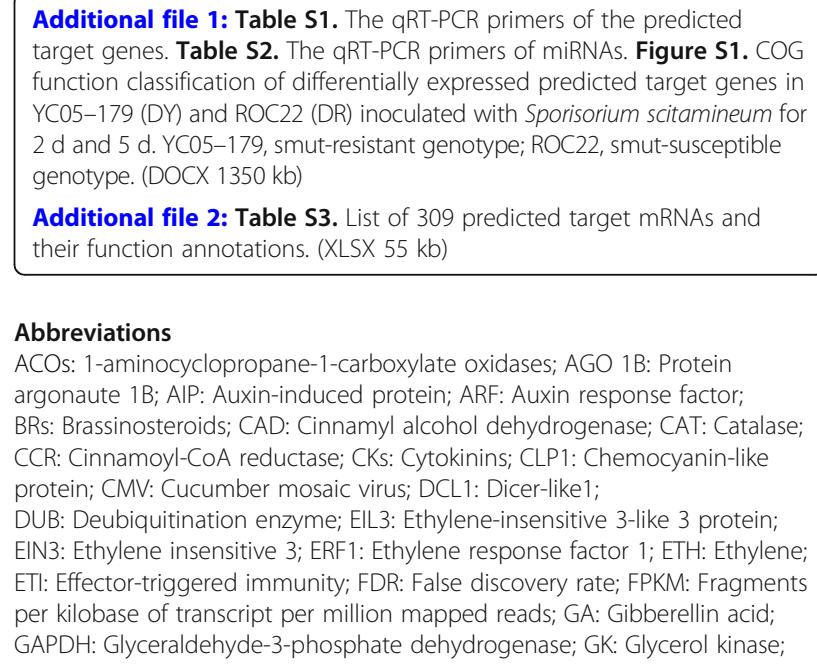

Additional file 2: Table S3. List of 309 predicted target mRNAs and their function annotations. (XLSX $55 \mathrm{~kb}$ )

\footnotetext{
Abbreviations

ACOs: 1-aminocyclopropane-1-carboxylate oxidases; AGO 1B: Protein argonaute 1B; AIP: Auxin-induced protein; ARF: Auxin response factor; BRs: Brassinosteroids; CAD: Cinnamyl alcohol dehydrogenase; CAT: Catalase; CCR: Cinnamoyl-CoA reductase; CKs: Cytokinins; CLP1: Chemocyanin-like protein; CMV: Cucumber mosaic virus; DCL1: Dicer-like1;

DUB: Deubiquitination enzyme; EIL3: Ethylene-insensitive 3-like 3 protein; EIN3: Ethylene insensitive 3; ERF1: Ethylene response factor 1; ETH: Ethylene; ETI: Effector-triggered immunity; FDR: False discovery rate; FPKM: Fragments per kilobase of transcript per million mapped reads; GA: Gibberellin acid;
} GAPDH: Glyceraldehyde-3-phosphate dehydrogenase; GK: Glycerol kinase; 
GO: Gene ontology; GRF8: Growth-regulating factor 8; HRs: Hypersensitive reactions; JA: Jasmonic acid; KEGG: Kyoto encyclopedia of genes and genomes; LHT1: Lysine histidine transporter 1; miRNAs: MicroRNAs; MLO: Mildew resistance locus o; MYB: v-myb avian myeloblastosis viral oncogene homolog; NAM: No apical meristem; NBT: Nitroblue tetrazolium; NHO1: Nonhost resistance 1; PAL: Phenylalanine ammonia lyase; PARE: Parallel analysis of RNA ends; PCD: Programmed cell death; PERK2: Proline-rich receptor-like protein kinase 2; POD: Peroxidase; PP2C: Protein phosphatase 2C; PPO: Polyphenol oxidase; PTGS: Post-transcriptional gene silencing; PVP: Polyvinylpyrrolidone: RISC: RNA-induced silencing complex; SA: Salicylic acid; SAMDC: S-adenosylmethionine decarboxylase; SAMS: S-adenosylmethionine synthetase; SAR: Systematic acquired resistance; SAUR: Small auxin up RNAs; SERK: Somatic embryogenesis receptor kinase; SOD: Superoxide dismutase; SPL: Squamosa promoter-binding-like protein; TAL: Tyrosine ammonia lyase; TAV: Tomato aspermy virus; UBC5: Ubiquitin-conjugating enzyme E2 5; UCH-L5: Ubiquitin carboxyl-terminal hydrolase isozyme L5-like

\section{Acknowledgements}

Not applicable.

\section{Funding}

This work was supported by Natural Science Foundation of Fujian province, China (2015 J06006), the National Natural Science Foundation of China (31501363, 31671752, 31101196, and 31340060), the Research Funds for Distinguished Young Scientists in Fujian Agriculture and Forestry University (xjq201630), the Program for New Century Excellent Talents in Fujian Province University (JA14095) and the earmarked fund for the Modern Agriculture Technology of China (CARS-17). The funding bodies had no role in the design of the study and collection, analysis, and interpretation of data and in writing the manuscript.

\section{Availability of data and materials}

The data supporting the conclusions of this article are within the paper and its additional files. All sequencing reads are deposited in the National Center for Biotechnology Information under the BioProject number PRJNA479686 with the Sequence Read Archive (SRA) study accession SRP152564.

\section{Authors' contributions}

YCS, XHX and YXQ conceived, designed and initiated the project. XHX prepared materials. XHX, HL, NH, FL, WHS, and YYZ performed experiments and contributed to data analysis and validation. YCS and YXQ drafted the manuscript. LPX, KM and YXQ helped to revise the manuscript. All authors read and approved the final manuscript.

\section{Ethics approval and consent to participate}

Not applicable. This is to confirm that no specific permits were needed for the described experiments, and this study did not involve any endangered or protected species.

\section{Consent for publication}

Not applicable.

\section{Competing interests}

The authors declare that they have no competing interests.

\section{Publisher's Note}

Springer Nature remains neutral with regard to jurisdictional claims in published maps and institutional affiliations.

\section{Author details}

'Key Laboratory of Sugarcane Biology and Genetic Breeding, Ministry of Agriculture, Fujian Agriculture and Forestry University, Fuzhou 350002, China. ${ }^{2}$ Key Laboratory of Ministry of Education for Genetics, Breeding and Multiple Utilization of Crops, College of Crop Science, Fujian Agriculture and Forestry University, Fuzhou 350002, China. ${ }^{3}$ Department of Genetics, Hazara University, Mansehra 21300, Pakistan.
Received: 21 July 2018 Accepted: 20 December 2018

Published online: 18 January 2019

\section{References}

1. Nzioki HS, Jamoza JE, Olweny CO, Rono JK. Characterization of physiologic races of sugarcane smut (Ustilago scitaminea) in Kenya. Afr J Microbiol Res. 2010:4:1694-7.

2. Singh N, Somai BM, Pillay D. Smut disease assessment by PCR and microscopy in inoculated tissue cultured sugarcane cultivars. Plant Sci. 2004; 167(5):987-94.

3. Sundar AR, Barnabas EL, Malathi P, Viswanathan R. A mini-review on smut disease of sugarcane caused by Sporisorium scitamineum. In: Mworia J, editor. Botany. Croatia: InTech Press; 2012. p. 109-28.

4. Chao CP, Hoy JW, Saxton AM, Martin FA. Heritability of resistance and repeatability of clone reactions to sugarcane smut in Louisiana. Phytopathology. 1990;80:622-6.

5. Que YX, Xu LP, Wu QB, Liu YF, Ling H, Liu YH, et al. Genome sequencing of Sporisorium scitamineum provides insights into the pathogenic mechanisms of sugarcane smut. BMC Genomics. 2014;15:996.

6. Taniguti LM, Schaker PDC, Benevenuto J, Peters LP, Carvalho G, Palhares A et al. Complete genome sequence of Sporisorium scitamineum and biotrophic interaction transcriptome with sugarcane. PLoS One. 2015;10 e0129318.

7. Dutheil JY, Gertrud M, Gabriel S, Christian MKS, Martin M, Ulrich G, et al. A tale of genome compartmentalization: the evolution of virulence clusters in smut fungi. Genome Biol Evol. 2016:8:681-704.

8. Xu LP, Chen RK. Identification of RAPD marker linked to smut resistance gene in sugarcane. Chin J Appl Environ Biol. 2004;10:263-7.

9. Gao YJ, Zhang RH, Zhang GM, Yang L, Duan WX, Wang ZP, et al. Screening of SSR marker linked to smut resistance gene in sugarcane. Chin J Trop Crop. 2013;34:2222-6.

10. Wu QB, Xu LP, Guo JL, Su YC, Que YX. Transcriptome profile analysis of sugarcane responses to Sporisorium scitaminea infection using solexa sequencing technology. Biomed Res Int. 2013;2013:298920.

11. Que YX, Su YC, Guo JL, Wu QB, Xu LP. A global view of transcriptome dynamics during Sporisorium scitamineum challenge in sugarcane by RNAseq. PLoS One. 2014;9:e106476.

12. Su YC, Xu LP, Wang ZQ, Peng Q, Yang YT, Chen Y, et al. Comparative proteomics reveals that central metabolism changes are associated with resistance against Sporisorium scitamineum in sugarcane. BMC Genomics. 2016;17:800

13. Que YX, Xu LP, Lin JW, Ruan MH, Zhang MQ, Chen RK. Differential protein expression in sugarcane during sugarcane-Sporisorium scitamineum interaction revealed by 2-DE and MALDI-TOF-TOF/MS. Comp Funct Genom. 2011;2011:989016.

14. Su YC, Zhang YY, Huang N, Liu F, Su WH, Xu LP, et al. Small RNA sequencing reveals a role for sugarcane miRNAs and their targets in response to Sporisorium scitamineum infection. BMC Genomics. 2017:18:325.

15. Zhang BH, Stellwag EJ, Pan XP. Large-scale genome analysis reveals unique features of microRNAs. Gene. 2009:443:100-9.

16. Lee RC, Feinbaum RL, Ambros V. The C. elegans heterochronic gene lin-4 encodes small RNAs with antisense complementarity to lin-14. Cell. 1993;75: 843-54.

17. Reinhart BJ, Weinstein EG, Rhoades MW, Bonnie B, Bartel DP. MicroRNAs in plants. Genes Dev. 2002:16:1616-26.

18. Xie ZX, Allen E, Fahlgren N, Calamar A, Givan SA, Carrington J. Expression of Arabidopsis MIRNA genes. Plant Physiol. 2005;138:2145-54.

19. Mica E, Gianfranceschi L, Pè ME. Characterization of five microRNA families in maize. J Exp B. 2006:57:2601-12.

20. Lewis $B P$, Burge $C B$, Bartel DP. Conserved seed pairing, often flanked by adenosines, indicates that thousands of human genes are microRNA targets. Cell. 2005;120:15-20.

21. Sunkar R, Zhu JK. Novel and stress-regulated microRNAs and other small RNAs from Arabidopsis. Plant Cell. 2004;16:2001-19.

22. Feng $\mathrm{H}$, Zhang Q, Wang QL, Wang XJ, Liu J, Li M, et al. Target of taemiR408, a chemocyanin-like protein gene (TaCLP1), plays positive roles in wheat response to high-salinity, heavy cupric stress and stripe rust. Plant Mol Biol. 2013:83:433-43.

23. Feng JL, Wang $Y W$, Lin RH, Chen JS. Altered expression of microRNAs and target mRNAs in tomato root and stem tissues upon different viral infection J Phytopathol. 2013;161:107-19. 
24. Zhang XH, Zou Z, Zhang JH, Zhang YY, Han QQ, Hu TX, et al. Overexpression of sly-miR156a in tomato results in multiple vegetative and reproductive trait alterations and partial phenocopy of the sft mutant. FEBS Lett. 2011;585:435-9.

25. Peng H. Cloning, identification and functional analysis of miRNAs under the infection of Maize R. solani AG1-1A. In: Sichuan Agricultural University master dissertation; 2009 .

26. Voinnet O. Origin, biogenesis, and activity of plant microRNAs. Cell. 2009; 136:669-87.

27. Jones-Rhoades MW, Bartel DP, Bartel B. MicroRNAs and their regulatory roles in plants. Annu Rev Plant Biol. 2006;57:19-53.

28. German MA, Pillay M, Jeong DH, Hetawal A, Luo S, Janardhanan P, et al. Global identification of microRNA-target RNA pairs by parallel analysis of RNA ends. Nat Biotechnol. 2008;26:941-6.

29. Addo-Quaye C, Eshoo TW, Bartel DP, Axtell MJ. Endogenous siRNA and miRNA targets identified by sequencing of the Arabidopsis degradome. Curr Biol. 2008:18:758-62

30. Mao L, Lin HJ, Jian G, Li WR, Shen Y, Zhao MJ, et al. Identification and characterization of differentially expressed microRNAs in response to Rhizoctonia solani, in maize. Acta Physiol Plant. 2015;37:250.

31. Zhao CZ, Xia H, Cao TJ, Yang Y, Zhao SZ, Hou L, et al. Small RNA and degradome deep sequencing reveals peanut microRNA roles in response to pathogen infection. Plant Mol Biol Rep. 2015;33:1013-29.

32. Gibson UE, Heid CA, Williams PM. A novel method for real time quantitative RT-PCR. Genome Res. 1996;6:995-1001.

33. Varkonyi-Gasic E, Wu R, Wood M, Walton EF, Hellens RP. Protocol: a highly sensitive RT-PCR method for detection and quantification of microRNAs. Plant Methods. 2007;3:12.

34. Yao W, Yu AL, Xu JS, Zhou H, Zhang MQ, Chen RK. A simple and quick method for extracting sugarcane genomic DNA. J Agric Biotechnol. 2005;13:121-2.

35. Su YC, Wang SS, Guo JL, Xue BT, Xu LP, Que YX. A TaqMan real-time PCR assay for detection and quantification of Sporisorium scitamineum in sugarcane. Scientific World J. 2013;2013:942682.

36. Madan KV, Namita S, Meena N, Solomon S, Agnihotri VP. Enzyme activity and cane genotype resistance to red rot. Sugar Cane. 1991;2:6-8.

37. Moerschbacher BM, Noll UM, Flott BE, Reisener HJ. Lignin biosynthetic enzymes in stem rust infected, resistant and susceptible near-isogenic wheat lines. Physiol Mol Plant P. 1988;33:33-46.

38. Giannopolitis CN, Ries SK. Superoxide dismutases I. occurrence in higher plants. Plant Physiol. 1977:59:309-14.

39. Beers RF, Sizer IW. A spectrophotometric method for measuring the breakdown of hydrogen peroxide by catalase. J Biol Chem. 1952;195:133-40

40. Galeazzi MAM, Sgarbieri VC, Constantinides SM. Isolation, purification and physicochemical characterization of polyphenoloxidases (PPO) from a dwarf variety of banana (Musa cavendishii L.). J Food Sci. 1981;46:150-5.

41. Green NE, Hadwiger LA, Graham SO. Phenylalanine ammonia-lyase, tyrosine ammonia-lyase, and lignin in wheat inoculated with Erysiphe graminis f. sp. tritici. Phytopathology. 1975;65:1071-4.

42. Kofalvi SA, Nassuth A. Influence of wheat streak mosaic virus infection on phenylpropanoid metabolism and the accumulation of phenolics and lignin in wheat. Physiol Mol Plant P. 1995;47:365-77.

43. German MA, Luo S, Schroth G, Meyers BC, Green PJ. Construction of parallel analysis of RNA ends (PARE) libraries for the study of cleaved miRNA targets and the RNA degradome. Nat Protoc. 2009;4:356-62.

44. Addo-Quaye C, Miller W, Axtell MJ. Cleaveland: a pipeline for using degradome data to find cleaved small RNA targets. Bioinformatics. 2009;25:130-45.

45. Cao X, Wu Z, Jiang FL, Zhou R, Yang Z. Identification of chilling stressresponsive tomato microRNAs and their target genes by high-throughput sequencing and degradome analysis. BMC Genomics. 2014;15:1130.

46. Fahlgren N, Carrington JC. miRNA target prediction in plants. Method Mol Biol. 2010:592:51-7.

47. Yang JH, Liu XY, Xu BC, Zhao N, Yang XD, Zhang MF. Identification of miRNAs and their targets using high-throughput sequencing and degradome analysis in cytoplasmic male-sterile and its maintainer fertile lines of Brassica juncea. BMC Genomics. 2013;14:9.

48. Trapnell C, Williams BA, Pertea G, Mortazavi A, Kwan G, van Baren MJ, et al. Transcript assembly and quantification by RNA-seq reveals unannotated transcripts and isoform switching during cell differentiation. Nat Biotechnol. 2010;28:511-5.

49. Kerr MK. Design considerations for efficient and effective microarray studies. Biometrics. 2003;59:822-8.
50. Benjamini Y, Hochberg Y. Controlling the false discovery rate: a practical and powerful approach to multiple testing. J Roy Stat Soc. 1995;57:289-300.

51. Ling $H, W u Q B$, Guo $J L, X u L P, Q$ Q YX. Comprehensive selection of reference genes for gene expression normalization in sugarcane by real time quantitative RT-PCR. PLoS One. 2014;9:e97469.

52. Chen C, Ridzon DA, Broomer AJ, Zhou Z, Lee DH, Nguyen JT, et al. Realtime quantification of microRNAs by stem-loop RT-PCR. Nucleic Acids Res. 2005;33:e179.

53. Thiebaut F, Rojas CA, Almeida KL, Grativol C, Domiciano GC, Lamb CR, et al. Regulation of miR319 during cold stress in sugarcane. Plant Cell Environ. 2012;35:502-12.

54. Livak KJ, Schmittgen TD. Analysis of relative gene expression data using real-time quantitative PCR and the $2^{-\Delta \Delta C T}$ method. Methods. 2001;25:402-8.

55. Bate NJ, Orr J, Ni W, Meromi A, Nadlerhassar T, Doerner PW, et al. Quantitative relationship between phenylalanine ammonia-lyase levels and phenylpropanoid accumulation in transgenic tobacco identifies a ratedetermining step in natural product synthesis. P Natl Acad Sci USA. 1994:91: 7608-12.

56. Li L, Steffens JC. Overexpression of polyphenol oxidase in transgenic tomato plants results in enhanced bacterial disease resistance. Planta. 2002;215:239-47.

57. Chen M, Bao H, Wu QM, Wang YW. Transcriptome-wide identification of miRNA targets under nitrogen deficiency in Populus tomentosa using degradome sequencing. Int J Mol Sci. 2015;16:13937-58.

58. Liu HJ, Qin C, Chen Z, Zuo T, Yang XR, Zhou HK, et al. Identification of miRNAs and their target genes in developing maize ears by combined small RNA and degradome sequencing. BMC Genomics. 2014;15:25.

59. Fukaki H, Taniguchi N, Tasaka M. PICKLE is required for SOLITARY-ROOT/ IAA14-mediated repression of ARF7 and ARF19 activity during Arabidopsis lateral root initiation. Plant J. 2006;48:380-9.

60. Tian CE, Muto H, Higuchi K, Matamura T, Tatematsu K, Koshiba T, et al. Disruption and overexpression of auxin response factor 8 gene of Arabidopsis affect hypocotyl elongation and root growth habit, indicating its possible involvement in auxin homeostasis in light condition. Plant J. 2004; 40:333-43.

61. Liu DM, Song Y, Chen ZX, Yu D. Ectopic expression of miR396 suppresses GRF target gene expression and alters leaf growth in Arabidopsis. Physiol Plantarum. 2009;136:223-36.

62. Bouche N, Yellin A, Snedden WA, Fromm H. Plant-specific calmodulinbinding proteins. Annu Rev Plant Biol. 2005;56:435-66.

63. Wolter M, Hollricher $K$, Salamini F, Schulze-Lefert $P$. The mlo resistance alleles to powdery mildew infection in barley trigger a developmentally controlled defence mimic phenotype. Mol Gen Genet. 1993;239:122-8.

64. Afrin S, Zhu J, Cao H, Huang J, Xiu H, Luo T, et al. Molecular cloning and expression profile of an abiotic stress and hormone responsive MYB transcription factor gene from Panax ginseng. Acta Bioch Bioph Sin. 2015;47: 267-77.

65. Lu M, Tang XY, Zhou JM. Arabidopsis NHO1 is required for general resistance against Pseudomonas bacteria. Plant Cell. 2001;13:437-47.

66. Kang L, Li JX, Zhao TH, Xiao FM, Tang XY, Thilmony R. Interplay of the Arabidopsis nonhost resistance gene NHO1 with bacterial virulence. P Natl Acad Sci USA. 2003;100:3519-24.

67. Yang XY, Wang LC, Yuan DJ, Lindsey K, Zhang XL. Small RNA and degradome sequencing reveal complex miRNA regulation during cotton somatic embryogenesis. J Exp Bot. 2013;64:1521-36.

68. Llave C, Xie ZX, Kasschau KD, Carrington JC. Cleavage of scarecrow-like mRNA targets directed by a class of Arabidopsis miRNA. Science. 2002;297:2053-6.

69. Ouellet DL, Perron MP, Gobeil LA, Plante P, Provost P. MicroRNAs in gene regulation: when the smallest governs it all. J Biomed Biotechnol. 2014; 2006:69616.

70. Wong J, Gao L, Yang Y, Zhai JX, Arikit S, Yu Y, et al. Roles of small RNAs in soybean defense against Phytophthora sojae infection. Plant J. 2014;79:928-40.

71. Harris MA, Clark J, Ireland A, Lomax J, Ashburner M, Foulger R, et al. The gene ontology (GO) database and informatics resource. Nucleic Acids Res. 2004;32:258-61.

72. Aoki-Kinoshita KF, Kanehisa M. Gene annotation and pathway mapping in KEGG. Method Mol Biol. 2007;396:71-92.

73. Coram TE, Settles ML, Chen XM. Transcriptome analysis of high-temperature adult-plant resistance conditioned by Yr39 during the wheat-Puccmia strifformis f. sp tritici interaction. Mol Plant Pathol. 2008;9:479-93.

74. Bolton MD, Kolmer JA, Xu WW, Garvin DF. Lr34-mediated leaf rust resistance in wheat: transcript profiling reveals a high energetic demand supported by 
transient recruitment of multiple metabolic pathways. Mol Plant Microbe In. 2008;21:1515-27.

75. Isaacson T, Kosma DK, Matas AJ, Buda GJ, He Y, Yu B, et al. Cutin deficiency in the tomato fruit cuticle consistently affects resistance to microbial infection and biomechanical properties, but not transpirational water loss. Plant J. 2009;60:363-77.

76. Kavitha M, Sundar AR, Padmanaban P, Viswanathan R, Malathi P. Comparative study on early detection of sugarcane smut (Sporisorium scitamineum) by polymerase chain reaction (PCR) and microscopy. Afr J Biotechnol. 2014;13:4635-8.

77. Battle M, Bender ML, Tans PP, White JW, Ellis JT, Conway T, et al. Global carbon sinks and their variability inferred from atmospheric $\mathrm{O}_{2}$ and $\delta^{13} \mathrm{C}$. Science. 2000;287:2467-70.

78. Andersen JR, Zein I, Wenzel G, Darnhofer B, Eder J, Ouzunova M, et al. Characterization of phenylpropanoid pathway genes within European maize (Zea mays L.) inbreds. BMC Plant Biol. 2008;8:147-59.

79. Miedes E, Vanholme R, Boerjan W, Molina A. The role of the secondary cell wall in plant resistance to pathogens. Front Plant Sci. 2015;5:358.

80. Leplé JC, Dauwe R, Morreel K, Storme V, Lapierre C, Pollet B, et al. Downregulation of cinnamoyl-coenzyme A reductase in poplar: multiplelevel phenotyping reveals effects on cell wall polymer metabolism and structure. Plant Cell. 2007;19:3669-91.

81. Eynck C, Séguin-swartz G, Clarke WE, Parkin IA. Monolignol biosynthesis is associated with resistance to Sclerotinia sclerotiorum in Camelina sativa. Mol Plant Pathol. 2012;13:887-99.

82. Hano C, Martin I, Fliniaux O, Legrand B, Gutierrez L, Arroo RRJ, et al. Pinoresinol-lariciresinol reductase gene expression and secoisolariciresinol diglucoside accumulation in developing flax (Linum usitatissimum) seeds. Planta. 2006;224:1291-301.

83. Mayer AM. Polyphenol oxidases in plants-recent progress. Phytochemistry. 1987:26:11-20.

84. Wing SS. Deubiquitinating enzymes-the importance of driving in reverse along the ubiquitin-proteasome pathway. Int J Biochem Cell B. 2003;35:590-605.

85. Xie DX, Feys BF, James S, Nieto-Rostro M, Turner JG. COl1: an Arabidopsis gene required for jasmonate-regulated defense and fertility. Science. 1998; 280:1091-4.

86. Boyle P, Le Su E, Rochon A, Shearer HL, Murmu J, Chu JY, et al. The BTB/POZ domain of the Arabidopsis disease resistance protein NPR1 interacts with the repression domain of TGA2 to negate its function. Plant Cell. 2009;21:3700-13.

87. Qiao H, Chang KN, Yazaki J, Ecker JR. Interplay between ethylene, ETP1/ETP2 F-box proteins, and degradation of EIN2 triggers ethylene responses in Arabidopsis. Genes Dev. 2009;23:512-21.

88. Zhao MZ, Wang QS, Lai WJ, Zhao XY, Shen HY, Nie FL, et al. In vitro bioactivity and biocompatibility evaluation of bulk nanostructured titanium in osteoblast-like cells by quantitative proteomic analysis. J Mater Chem B. 2013;1:1926-38.

89. Tanaka K, Suzuki T, Hattori N, Mizuno Y. Ubiquitin, proteasome and parkin. BBA Mol Cell Res. 2004;1695:235-47.

90. Hurley JH, Faber HR, Worthylake D, Meadow ND, Roseman S, Pettigrew DW et al. Structure of the regulatory complex of Escherichia coli IIIGlc with glycerol kinase. Science. 1993;259:673-7.

91. Chen HL, Wang YQ, Chu CC, Li P. Plant non-host resistance: current progress and future prospect. Hereditas. 2008;30:977-82.

92. Gao ZL, Xiao XR, Chen Y, Li XQ, Fu XM, Lin DZ, et al. Isolation and expression analysis of rice OsNHO1 gene. Chin J Trop Crop. 2014;5:30-5.

93. Greenberg JT, Guo A, Klessig DF, Ausubel FM. Programmed cell death in plants: a pathogen-triggered response activated coordinately with multiple defense functions. Cell. 1994;77:551-63.

94. Dietrich RA, Delaney TP, Uknes SJ, Ward ER, Ryals JA, Dangl JL, et al. Arabidopsis mutants simulating disease resistance response. Cell. 1994;77:565-77.

95. Xu HM, Liu YH, Wang JM, Tian BM, Wang PT. Cloning and expression analysis of Mlo after Blumeria gramins f. sp. tritici infection in wheat. J Triticeae Crop. 2010;30:401-5.

96. Luo Z, Zhang JZ, Hu DW. Cytological examination on interactions between Mlo near-isogenic lines of barley and their leaf blight pathogen, Alternaria tenuissima. Acta Phytopathol Sin. 2009;39:36-42.

97. Bari R, Jones JDG. Role of plant hormones in plant defence responses. Plant Mol Biol. 2009;69:473-88.

98. Khatabi B, Molitor A, Lindermayr C, Pfiffi S, Durner J, Von DW, et al. Ethylene supports colonization of plant roots by the mutualistic fungus Piriformospora indica. PLoS One. 2012;7:e35502.
99. Thomma BPHJ, Eggermont K, Tierens KFMJ, Broekaert WF. Requirement of functional ethylene-insensitive 2 gene for efficient resistance of Arabidopsis to infection by Botrytis cinerea. Plant Physiol. 1999;121:1093-101.

100. Gee MA, Hagen G, Guilfoyle TJ. Tissue-specific and organ-specific expression of soybean auxin-responsive transcripts GH3 and SAURs. Plant Cell. 1991;3: 419-30.

101. Arif SA, Taylor MA, George LA, Butler AR, Burch LR, Davies HV, et al. Characterisation of the S-adenosylmethionine decarboxylase (SAMDC) gene of potato. Plant Mol Biol. 1994;26:327-38.

102. Takizawa R, Nishizawa NK, Nakanishi H, Mori S. Effect of iron deficiency on S-adenosylmethionine synthetase in barley roots. J Plant Nutr. 1996;19: 1189-200.

103. Waie B, Rajam MV. Effect of increased polyamine biosynthesis on stress responses in transgenic tobacco by introduction of human Sadenosylmethionine gene. Plant Sci. 2003;164:727-34.

104. Song XP, Zhang BQ, Huang X, Yang LT, Li YR. Cloning and expression of sugarcane S-adenosylmethionine synthetase gene SCSAM. Acta Agron Sin. 2014;40:1002-10.

105. Yang S, Sweetman JP, Amirsadeghi S, Barghchi M, Huttly AK, Chung WI, et al. Novel anther-specific myb genes from tobacco as putative regulators of phenylalanine ammonia-lyase expression. Plant Physiol. 2001;126:1738-53.

106. Rawat R, Schwartz J, Jones MA, Sairanen I, Cheng YF, Andersson CR, et al. REVEILLE1, a Myb-like transcription factor, integrates the circadian clock and auxin pathways. P Natl Acad Sci USA. 2009;106:16883-8.

107. Mengiste T, Chen X, Salmeron J, Dietrich R. The botrytis susceptible 1 gene encodes an R2R3MYB transcription factor protein that is required for biotic and abiotic stress responses in Arabidopsis. Plant Cell. 2003;15:2551-65.

108. Daniel X, Lacomme C, Morel JB, Roby D. A novel myb oncogene homologue in Arabidopsis thaliana related to hypersensitive cell death. Plant J. 1999;20:57-66.

109. Yang Y, Klessig DF. Isolation and characterization of a tobacco mosaic virusinducible myb oncogene homolog from tobacco. P Natl Acad Sci USA. 1996;93:14972-7.

110. Xie ZX, Kasschau KD, Carrington JC. Negative feedback regulation of dicerlike1 in Arabidopsis by microRNA-guided mRNA degradation. Curr Biol. 2003; 13:784-9.

111. Vaucheret $\mathrm{H}$, Mallory AC, Bartel DP. AGO1 homeostasis entails coexpression of MIR168 and AGO1 and preferential stabilization of miR168 by AGO1. Mol Cell. 2006;22:129-36.

\section{Ready to submit your research? Choose BMC and benefit from:}

- fast, convenient online submission

- thorough peer review by experienced researchers in your field

- rapid publication on acceptance

- support for research data, including large and complex data types

- gold Open Access which fosters wider collaboration and increased citations

- maximum visibility for your research: over $100 \mathrm{M}$ website views per year

At BMC, research is always in progress.

Learn more biomedcentral.com/submissions 